\title{
Interplay of Structure and Dynamics in
}

\section{Lithium/Ionic Liquid Electrolytes:}

\section{Experiment and Molecular Simulation}

Patrick Judeinstein ${ }^{1,2, *}$, Mehdi Zeghal $^{2}$, Doru Constantin ${ }^{2}$, Cristina Iojoiu $^{3}$, Benoit Coasne ${ }^{4, *}$

${ }^{1}$ Université Paris-Saclay, CEA, CNRS, LLB, 91191, Gif-sur-Yvette, France.

${ }^{2}$ Université Paris-Saclay, CNRS, Laboratoire de Physique des Solides, 91405, Orsay, France.

${ }^{3}$ Univ. Grenoble Alpes, Univ. Savoie Mont Blanc, CNRS, Grenoble INP, LEPMI, 38000 Grenoble, France

${ }^{4}$ Univ. Grenoble Alpes, CNRS, LIPhy, 38000 Grenoble, France

* To whom correspondence should be sent. Patrick Judeinstein (patrick.judeinstein@cea.fr), Benoit Coasne (benoit.coasne@univ-grenoble-alpes.fr) 


\begin{abstract}
Despite their promising use in electrochemical and electrokinetic devices, ionic-liquid based electrolytes often exhibit complex behavior arising from a subtle interplay of their structure and dynamics. Here, we report a joint experimental and molecular simulation study of such electrolytes obtained by mixing 1-butyl 3-methylimidazolium tetrafluoroborate with lithium tetrafluoroborate. More in detail, experiments consisting of X-ray scattering, pulsed field gradient NMR, and complex impedance spectroscopy are analyzed in the light of molecular dynamics simulations to probe the structural, dynamical and electrochemical properties of this ionic-liquid based electrolyte. Lithium addition promotes the nanostructuration of the liquid as evidenced from the appearance of a scattering prepeak that becomes more pronounced. Microscopically, using the partial structure factors determined from molecular dynamics, this prepeak is shown to correspond to the formation of well-ordered positive/negative charge series but also large aggregates $\left(\mathrm{Li}_{\mathrm{n}}\left(\mathrm{BF}_{4}\right)_{4-\mathrm{m}}\right)^{(4-\mathrm{m}+\mathrm{n})-}$ which develop upon lithium addition. Such nanoscale ordering entails a drastic decrease in both the molecular mobility and ionic conductivity. In particular, the marked association of $\mathrm{Li}^{+}$cations with four $\mathrm{BF}_{4}^{-}$anions and long ion pairing times, which are promoted upon lithium addition, are found to severely hinders the $\mathrm{Li}^{+}$transport properties.
\end{abstract}

\title{
Keywords
}

Ionic liquids and electrolytes; structure and dynamics; ionic conductivity; molecular dynamics

\section{TOC Graphic}

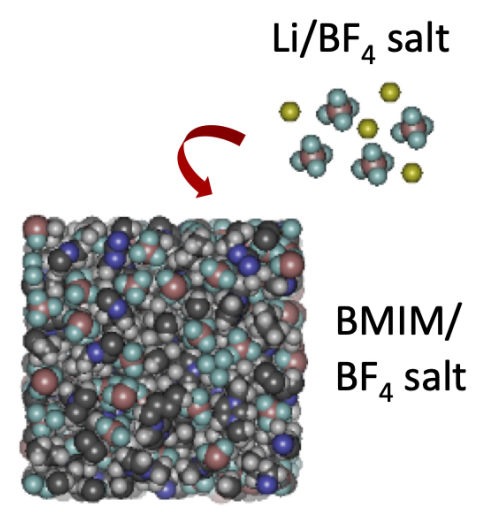




\section{Introduction}

Most common salts are made up of inorganic ions leading to melting points much above room temperature (e.g. $\mathrm{NaCl}$ melting point is $801^{\circ} \mathrm{C}$ ). In contrast, ionic liquids (ILs), which are obtained by combining organic cations and organic/inorganic anions, display melting points below $100^{\circ} \mathrm{C}$ or even below room temperature; the latter being coined as room temperature ionic liquids (RTILs). ${ }^{1}$ Such low melting points are a direct consequence of the cation molecular architecture which involves a delocalized electrostatic charge linked to a flexible organic moiety (in contrast, the anion is either a simple species such as a halogen or a more complex species such as $\mathrm{BF}_{4}, \mathrm{PF}_{6}, \mathrm{~N}\left(\mathrm{CF}_{3} \mathrm{SO}_{3}\right)_{2}$, etc. $){ }^{2}$ As a result, the subtle balance between ion attractive forces and segmental mobility explains the liquid character of these salts even at temperatures where the thermal energy $\mathrm{k}_{\mathrm{B}} \mathrm{T}$ is much smaller than the characteristic Coulomb interaction. The chemical bonding between the two conflicting moieties ${ }^{3,4}$ - i.e. charged center and flexible component of the cation - also induces some local segregation leading to self-organization inside the ionic liquid phase. This nanoscale organization is experimentally evidenced by the presence of a first sharp diffraction peak (also called prepeak) in the wide/small angle X-ray/neutron scattering experiments. ${ }^{5,6}$ Typically, this prepeak falls in the range $Q \sim 0.3-0.7 \AA^{-1}$ corresponding to a correlation distance $l \sim 2 \pi / \mathrm{Q}$ between 0.9 and $2.1 \mathrm{~nm}$. It was recently reported that such nanoscale organization has a strong impact on the dynamical properties inside these compounds. ${ }^{7}$ In particular, the detailed molecular arrangement resulting from Coulomb interactions between positive and negative charges combined with ion specific van der Waals and steric interactions leads to unanswered questions regarding the ion pairing mechanisms and the ionic strength in these electrolytes. ${ }^{8}$

It is estimated that millions of ionic liquids compositions can be achieved because of the huge number of cations and anions that can be combined. Such a nearly infinite versatility has raised significant interest in the physical chemistry community as this allows - at least in principle tuning their properties at will (viscosity, density, conductivity, etc.)..$^{9,10,11}$ In particular, because 
they are liquids in a broad range of temperatures, many applications can be envisaged in which these salts are used as solvent; tuning their structure allows one to dissolve more selectively apolar or polar molecules and even salts up to high concentrations. Moreover, owing to their low flammability as well as good thermal and electrochemical stabilities, solutions of alkali or alkaliearth salts in ionic liquids are also considered as promising electrolytes for batteries ${ }^{12,13}$ despite the following weaknesses: (1) their rather low ionic conductivity compared to solution of alkali salts in electrochemically suitable solvents (e.g. carbonates) and (2) a deceptively low transference number of the alkali cation. There is already strong experimental evidence based on multitechnique/multiscale approaches pointing to a strong coupling between these structural and dynamical peculiarities. Yet, key microscopic information such as data about ion coordination shell and ion pairing lifetime is still lacking because these observables are very difficult to access experimentally (despite indirect probing techniques available such as nuclear Overhauser effect in $\mathrm{NMR}^{14,15,16,17,18}$, $\operatorname{Raman}^{19,20}$ or infrared spectroscopy ${ }^{21}$ or from X-Ray structural determination in the solid state ${ }^{22}$ ).

In this context, classical molecular dynamics (MD) is a powerful complementary approach as it allows describing at the microscopic scale the structure and dynamics of such ion assemblies. Typically, motion modes from molecular vibrations up to self-diffusion processes can be probed from $10^{-15} \mathrm{~s}$ up to $10^{-7} \mathrm{~s}$ while geometry/coordination shell up to local nanostructure can be explored on length scales from $10^{-2}$ to a few $\mathrm{nm}$. Time/space information such as ion correlation modes and ion pair lifetime can be also estimated from MD trajectories. ${ }^{23,24}$ However, to ensure that meaningful information is obtained at such level, it is mandatory to select a robust and accurate force field combined with appropriate analysis methods and approximations..$^{25,26}$ In the specific context of ILs, many approaches and force fields are available with a satisfactory match between experimental and simulated data. ${ }^{27,28,29}$ This has led to a boost in molecular simulation study on these electrolytes for electrochemical systems (i.e batteries and supercapacitors). In particular, such molecular tools appear as well suited to investigate electrolytes made up of 
lithium salt dissolved in ionic liquid..$^{30,31,32,33,34}$ Of particular relevance, molecular dynamics can be used to unravel the motion modes of the $\mathrm{Li}^{+}$cation and its association with other ions ${ }^{35,36}$ which are essential to tune ionic conductivity as well as coordination sphere modifications at the active electrodes and during electrochemical processes. ${ }^{37,38,39}$

In this paper, we report a joint experimental and molecular simulation study on the intermediate structural and dynamical range $(0.1-10 \mathrm{~nm} / 0.001-100 \mathrm{~ns})$ of an ionic liquidbased electrolyte containing lithium ions: a liquid mixture of 1-butyl 3-methyl imidazolium tetrafluoroborate $\left(\mathrm{BMIMBF}_{4}\right)$ and lithium tetrafluoroborate $\left(\mathrm{LiBF}_{4}\right)$ salts. This work aims at providing a microscopic description of the molecular structural and dynamical mechanisms for all different species inside the ionic liquid electrolyte including ion association. It also intends to gain insights into the nanostructuration of the ionic liquid and the influence of replacing a large, flexible and charge delocalized cation $\left(\mathrm{BMIM}^{+}\right)$by a small cation $\left(\mathrm{Li}^{+}\right)$with low polarizability. More in detail, as illustrated in Fig. 1, we focus on the properties of electrolytes obtained by dissolving a lithium salt, $\mathrm{LiBF}_{4}$, into an ionic liquid, $\mathrm{BMIMBF}_{4}$. These electrolytes are probably not optimal candidates for advanced battery technologies and electrochemical systems; in particular, we are aware of the reduced stabilty of the $\mathrm{BF}_{4}{ }^{-}$anion, the strong viscosity of these systems, their high hydrophilicity, and relatively poor ionic conductivity. Yet, they can be regarded as prototypical systems to study the behavior of such materials because both the cations and anions have simple geometries (among those leading to RTILs) and large amounts of Li cations can be introduced. In practice, an experimental multitechnique approach is combined with an all-atom molecular simulation method to probe the structure and dynamics of these electrolytes. On the one hand, experimentally, their structure is probed using wide angle X-ray scattering (WAXS) while their self-diffusivity and ionic conductivity are investigated using pulsed field gradient NMR (PFG-NMR) and impedance spectroscopy (EIS), respectively. On the other hand, molecular dynamics is used to probe the same properties and gain insights into the microscopic mechanisms responsible for the structure and dynamics of these ionic liquid-based 
electrolytes containing Li ions. The experimental and molecular dynamics results are compared for $\mathrm{BMIMBF}_{4}+\mathrm{LiBF}_{4}$ systems with a broad composition range: $0 \leq \mathrm{Li} / \mathrm{BMIM} \leq 0.5$ (throughout this paper, the mole ratio $f=[\mathrm{Li} / \mathrm{BMIM}]$ is used to distinguish the different systems under study).
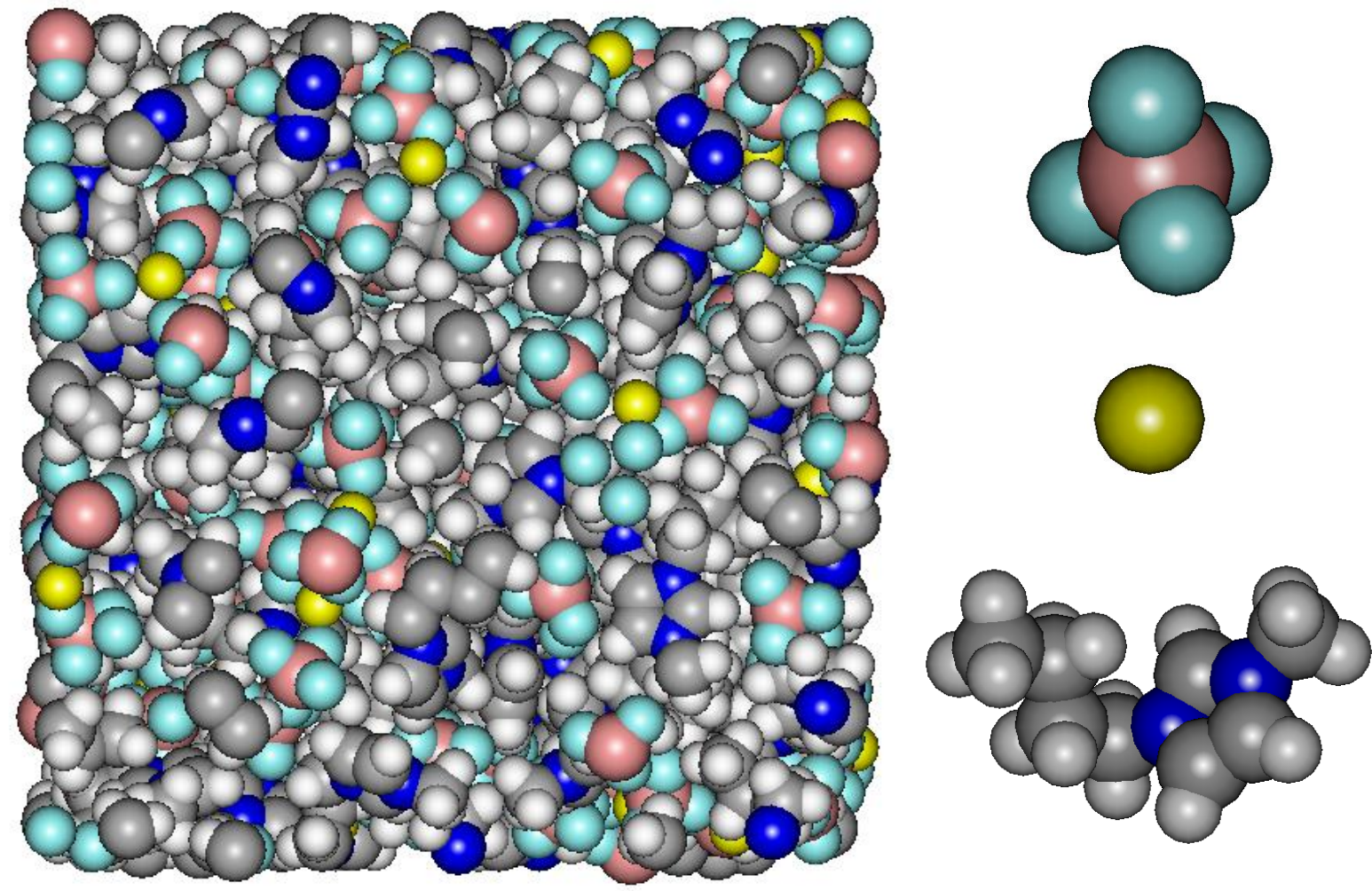

Fig. 1. Typical molecular configuration at $T=500 \mathrm{~K}$ of an ionic liquid-based electrolyte made up of BMIM cations and $\mathrm{BF}_{4}$ anions containing a fraction $f=[\mathrm{Li} / \mathrm{BMIM}]$ of $\mathrm{Li}^{+}$cations (here, $f=$ $0.5)$. The box size in each direction is $L \sim 35 \AA$. Each ion type is shown on the right: (top) $\mathrm{BF}_{4}$, (middle) Li, and (bottom) BMIM. The cyan and magenta spheres are the F and B atoms in the $\mathrm{BF}_{4}$ anion, the yellow sphere the Li cation, and the grey, white and blue spheres the $\mathrm{C}, \mathrm{H}$, and $\mathrm{N}$ atoms in the BMIM cation.

First, the ability of our MD approach to reproduce fairly well the experimental results in terms of dynamical and structural properties is discussed. Second, the experimental data are analyzed in the light of our molecular dynamics simulations to decipher the origin of the 
preapeak in IL scattering curves but also to identify the slow diffusion mechanisms of the lithium cation inside these mixtures.

\section{Methods}

\subsection{Experimental methods}

Material preparation. 1-butyl-3-methylimidazolium tetrafluoroborate $\left(\mathrm{BMIMBF}_{4}\right)$ and lithium tetrafluoroborate $\left(\mathrm{LiBF}_{4}\right)$ were provided by Aldrich and used as received. The electrolytes considered in this study were obtained by weighting and mixing defined amounts of $\mathrm{LiBF}_{4}$ and $\mathrm{BMIMBF}_{4}$. Dissolution was obtained under vigorous stirring at $70^{\circ} \mathrm{C}$ for 24 hours. The solutions were then dried under vacuum $\left(10^{-1} \mathrm{mmHg}, 60^{\circ} \mathrm{C}, 24 \mathrm{~h}\right)$. In this paper, the composition of the different electrolytes is described using the mole ratio $f$. Data will be reported for $f=0,0.1,0.3$ and 0.5 as the apparent solubility limit was observed around $f \sim 0.6-0.7$.

\section{Pulsed Field Gradient Nuclear Magnetic Resonance. Pulsed Field Gradient NMR (PFG-NMR)} measurements were performed on a 9.4 T spectrometer developed at Laboratoire de Physique des Solides, equipped with a Doty $5 \mathrm{~mm}$ diffusion probe with a $z$-axis gradient and a temperature controller. NMR resonance frequencies are 400.1 MHz, 376.5 MHz and $155.5 \mathrm{MHz}$ for ${ }^{1} \mathrm{H},{ }^{19} \mathrm{~F}$ and ${ }^{7} \mathrm{Li}$ nuclei, respectively. $4 \mathrm{~mm}$ NMR tubes, used to avoid convection phenomena, were sealed after filling to avoid any water uptake. The self-diffusion measurements were performed with a stimulated echo sequence. ${ }^{40}$ The gradient pulse duration, $\delta$ was set between $1 \mathrm{~ms}$ and $3 \mathrm{~ms}$ while the diffusion delay $\Delta$ was set at $25 \mathrm{~ms}$. Gradient strengths up to $1200 \mathrm{G} / \mathrm{cm}$ were used to record the data. The absence of convection in the samples of lower viscosity was checked by verifying that similar diffusion coefficients were obtained when using longer $\Delta$ (100ms). These experimental conditions enable the spin echo attenuation over a range of at least 1 decade, leading to a good accuracy $(<10 \%)$ in the self-diffusion coefficient determination. Typically, the self-diffusivity $D_{\mathrm{s}}$ was determined from the classical relationship:

$$
\ln \left(I / I_{0}\right)=-D_{s} G^{2} \gamma^{2} \delta^{2}(\Delta-\delta / 3)
$$


where $G$ is the magnitude of the gradient pulses and $\gamma$ the gyromagnetic ratio of the nucleus under investigation. $I$ and $I_{0}$ are the area of the signal obtained with and without gradient pulses, respectively.

Complex impedance spectroscopy. Ionic conductivities were determined by means of electrochemical impedance spectroscopy (EIS) using an HP 4192A Impedance Analyzer in the frequency range $5 \mathrm{~Hz}-1 \mathrm{MHz}$. The samples were placed in a dip-type glass cell with the measuring probe consisting of two Pt electrodes fixed at a constant distance. Cell constants were determined using a $0.1 \mathrm{M} \mathrm{KCl}$ solution. Measurements were performed under argon in the temperature range $25^{\circ} \mathrm{C}-80^{\circ} \mathrm{C}$ during heating and cooling ramp (to probe the reproduciblity of the measurements). The temperature was equilibrated at each step for $30 \mathrm{~min}$ prior to each measurement.

Wide-angle X-ray scattering. Wide-angle diffraction X-Ray scattering (WAXS) patterns were measured on the MOMAC LPS laboratory instrument at the Mo $K_{\alpha}$ wavelength $(0.711 \AA)$ in the $Q$ scattering vector range $0.1 \AA^{-1}<Q<3.2 \AA^{-1}$. A detailed description of the experimental setup can be found in Ref. ${ }^{41} ; 2$ mm capillary tubes were used to record the scattering patterns. The raw scattering signal was subtracted from the signal of an empty capillary tube.

\subsection{Computational Methods}

The structure and dynamics of the $\mathrm{LiBF}_{4} / \mathrm{BMIMBF}_{4}$ electrolytes were simulated by means of classical Molecular Dynamics (MD) using an all-atom description. All molecular simulations were performed using LAMMPS package. ${ }^{42}$ The classical force field developed by Canongia Lopes et al. ${ }^{43}$ with its new parameterization by Köddermann et al. ${ }^{26}$ was used for BMIM cations. The parameters by $\mathrm{Wu}$ et al. ${ }^{44}$ were used for $\mathrm{BF}_{4}^{-}$anions and those by Jensen et al. ${ }^{45}$ for $\mathrm{Li}^{+}$ cations. In all these force fields, the overall cation and anion charge is unity $(q=+/-1)$ with each 
atom $\mathrm{X}$ in the ion carrying a partial charge $\left(\left|q_{\mathrm{X}}\right| \leq 1\right)$ (Table 1 in the Supporting Information). Interactions between the different atoms were calculated as the sum of the dispersion interaction with a repulsive short-range contribution and the Coulombic interaction. The repulsiondispersion interactions were described using a Lennard-Jones potential in which the cross parameters $\sigma_{k j}$ and $\varepsilon_{k j}$ between atom types $k$ and $j$ are obtained from the Lorentz-Berthelot mixing rules of like-atom pairs. The like-atom parameters for the IL were taken from Ref. ${ }^{46}$. The electrostatic interactions were calculated using the Ewald sum technique to correct for the finite size of the simulation box (the parameters were chosen so that the accuracy is $10^{-5}$ ). More details are provided in the supplementary material (Table S1 and Figure S1).

Simulation boxes containing 124 ion pairs (box size $L \sim 35 \AA$ ) were used to determine several quantities such as dynamical parameters (self-diffusion coefficients, ionic conductivity). In addition, much larger systems containing 3348 ion pairs (box size $L \sim 100 \AA$ ) were also considered for the structural studies as they allow probing characteristic sizes larger than the observed heterogeneities. In particular, such large system sizes allow probing length scales compatible with the scattering vector range measured using WAXS experiments $\left(0.1 \AA^{-1}<Q<\right.$ $3.2 \AA^{-1}$ corresponding approximatively to distances in the range $2 \AA<r<60 \AA$ ). The temperature and pressure were controlled using a Nosé-Hoover thermostat and barostat (relaxation time parameter: 0.01 and $0.1 \mathrm{ps}$, respectively). ${ }^{47}$ The simulations were integrated using the Verlet algorithm with a timestep of $1 \mathrm{fs}$. Sampling times were set to $10 \mathrm{fs}$ and $1000 \mathrm{fs}$ to get short and long trajectory data, respectively. For all these calculations, a first 1 ns equilibration run was performed in the NPT ensemble $(P=1 \mathrm{bar}, T=500 \mathrm{~K})$ to bring the system to equilibrium. The use of higher temperature compared to experiments will be discussed in detail at the beginning of the next section. Then, another NVT equilibration run $(\sim 1 \mathrm{~ns})$ was performed before acquiring data in a final production run. For the short dynamical trajectories used to obtain very accurate mean square displacements at short times, $0.1 \mathrm{~ns}$ trajectories were recorded with a 10 fs sampling timestep. For longer dynamics, up to 200 ns trajectories with a 1 
ps sampling timestep were used. For the larger boxes used for the structural study, 50 configurations were used in the NPT ensemble and a large sampling timestep of 1 ps was considered to ensure that the different configurations are statistically uncorrelated.

\section{Results}

The aim of this section is to compare real world versus in silico experiments. To do this, the objective is to establish that an adequate molecular simulation methodology allows retrieving most semi-quantitative aspects of the experimental findings (both in terms of structural and dynamical quantities). It should be recalled that all MD calculations were performed at a larger temperature $(T=500 \mathrm{~K})$ in order to reach a Fickian diffusion regime with a decent trajectory duration (Fig S2) following previous works. ${ }^{38,48}$ Even if some ionic liquid MD studies are already performed at $300 \mathrm{~K},{ }^{49}$ strong ionic correlations between ions with high charge density $\left(\mathrm{BF}_{4}^{-}, \mathrm{Li}^{+}\right)$lead to liquid with strong interionic correlations and longer trajectories and/or higher temperatures are required to obtain valuable results. ${ }^{32,50}$ This is important to note that this trick necessarily affects the quantitative comparison established between our experimental and simulation approaches. Yet, in practice, this does not affect the qualitative picture that emerges from the comparison between the experimental and simulation datasets. Such temperature shifts between experiments and molecular dynamics are not unusual in such comparative studies..$^{29,51,52}$

\subsection{Structure factor $S(Q)$}

Fig. 2 presents the experimental X-Ray scattering data for $\mathrm{BMIMBF}_{4} / \mathrm{LiBF}_{4}$ electrolytes in the range $0.1 \AA^{-1}<Q<3.0 \AA^{-1}$ which corresponds to typical intermolecular correlation distances. In the case of ionic liquids, this scattering vector range allows probing the nanoscale structuration which results from the competition between coulombic ordering and entropic disordering forces. ${ }^{2,6}$ Fig. 2 also presents the total scattering function $S(Q)$ of $\mathrm{BMIMBF}_{4} / \mathrm{LiBF}_{4}$ electrolytes (from $f=0$ to 0.5 ) as calculated from molecular dynamics. These molecular simulation data are fully consistent with the experimental data, therefore indicating that our MD approach is a 
reasonable strategy to decipher structural aspects. For all compositions, two scattering peaks are observed; the strongest peak around $Q=1.5 \AA^{-1}$ is very weakly composition-dependent while the smallest peak around $Q=0.5 \AA^{-1}$ significantly depends on composition.

The strong and broad peak around $Q=1.5 \AA^{-1}$, which is observed in all ionic liquids, is known to correspond to inter- and intra-molecular contributions mainly related to neighboring molecules. ${ }^{2,7,53,54}$ This peak is slightly shifted towards larger $Q$ upon $\mathrm{Li}^{+}$salt addition $\left(1.52 \AA^{-1}\right.$ for $f=0$ and $1.57 \AA^{-1}$ for $\left.f=0.5\right)$ without modification of its shape and intensity. This contrasts with what is observed in other systems such as LiTFSI/Pyrrolidinium TFSI systems where a shift towards smaller $Q$ was measured upon Li ion addition (this effect being related to the size of Li(TFSI $)^{2-}$ anion $\left.^{55}\right)$. As already mentioned, the smaller peak at smaller $Q$ is very sensitive to the amount of Li salt being added to the electrolyte. It appears as a shoulder around $Q=0.55 \AA^{-1}$ for pure $\mathrm{BMIMBF}_{4}$, and as a peak around $0.46-0.47 \AA^{-1}$ when Li salt is added. This peak becomes more pronounced upon increasing the Li content (while its position remains nearly constant). In pure ionic liquid, this peak observed in the low $Q$ domain is sometimes referred to as prepeak (or first sharp diffraction peak). Such a low $Q$ peak is often measured in pure ionic liquid when a long alkyl chain is linked to the ionic moiety (e.g. imidazolium). It is a typical feature of the selforganization corresponding to a real space distance $d \sim 2 \pi / Q$ in the $1-2 \mathrm{~nm}$ range (a distance comparable to the size of the cation which is about $0.7-1 \mathrm{~nm}$ depending on its configuration). This correlation peak, which is therefore representative of the size of the heterogeneous nanodomains in the ionic liquid, also indicates that this repetition structure occurs over few domains (i.e. the liquid is rather well-organized and the anion coordination shell well-defined). This allowed some authors to propose structural models where the nanoscale structuration of the liquid is the consequence of strong segregation between polar and apolar moieties. ${ }^{67,49}$ To our knowledge, this is the first evidence of structural IL reorganization upon salt addition. In previously published studies,,$^{31,56}$ the effect of $\mathrm{LiBF}_{4}$ salt addition inside IL was depicted only 
from an MD approach to investigate only the short range organization (first coordination layer) from the analysis of radial distribution functions $G(r)$.

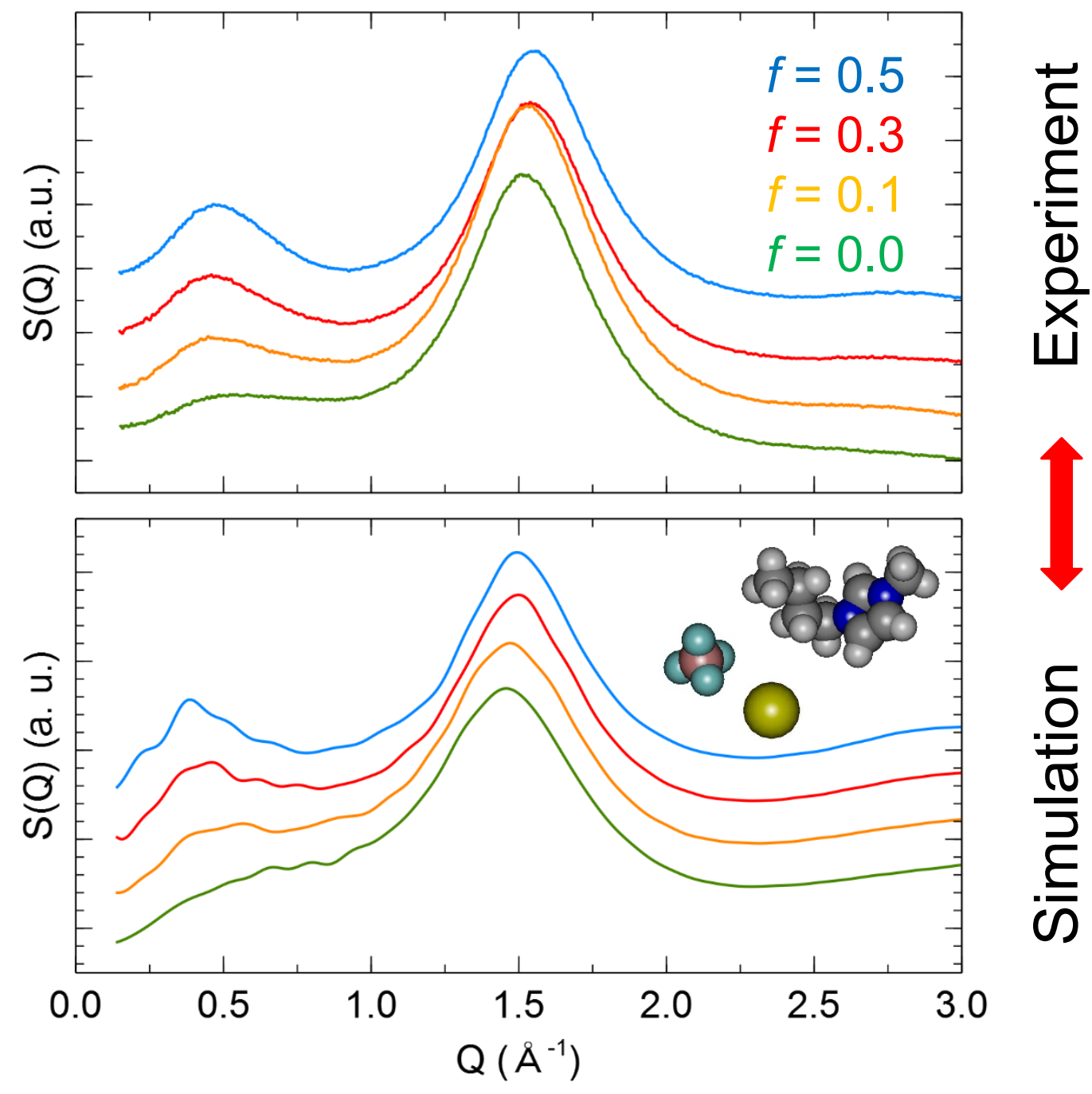

Fig. 2. WAXS diffraction patterns at room temperature for $\mathrm{BMIMBF}_{4} / \mathrm{LiBF}_{4}$ electrolytes with $0 \leq f \leq 0.5(f$, which is indicated in the graph, denotes the mole fraction of lithium dissolved into the ionic liquid): (top) Experiments at room temperature and (bottom) molecular dynamics at $T=$ $500 \mathrm{~K}$. Note that the curves for the different $f$ values have been shifted up for the sake of clarity. 


\subsection{Self-diffusion coefficient $D_{\mathrm{s}}$}

The self-diffusion coefficients $D_{\text {s }}$ of lithium cation $\left(\mathrm{Li}^{+}\right)$, imidazolium cation $\left(\mathrm{BMIM}^{+}\right)$and tetrafluoroborate anion $\left(\mathrm{BF}_{4}^{-}\right)$were measured experimentally by means of ${ }^{7} \mathrm{Li},{ }^{1} \mathrm{H}$ and ${ }^{19} \mathrm{~F}$ PFGNMR experiments respectively. In parallel, molecular dynamics simulations were performed to estimate numerically the self-diffusivity $D_{\mathrm{s}}$ of these different species. As shown in Fig. 3, both the experimental and simulated self-diffusivities as a function of the Li content in the ionic liquid-based electrolyte follow the same qualitative behavior. Without Li salt addition, the selfdiffusion coefficients obtained here are comparable to those already published for $\mathrm{BMIMBF}_{4}$ using PFG-NMR ${ }^{57,58}$ or field cycling NMR relaxometry ${ }^{59}$ measurements. These data show that the cation diffusion coefficient is close to that for the anion $\left(1.5 \times 10^{-11} \mathrm{vs} .1 .4 \times 10^{-11} \mathrm{~m}^{2} / \mathrm{s}\right)$. Upon Li salt addition, a strong decrease in the self-diffusion coefficient is observed for all ions as the apparent viscosity of the system increases (not measured in this work). This mobility decrease is more pronounced for the anion than the cation.

Moreover, despite its small mass, the self-diffusion of the $\mathrm{Li}^{+}$ion is slower than for the other ionic species. This behavior, which was already reported ${ }^{14,31,60}$ for several other lithium electrolytes based on ionic liquid, was shown to be related to ion pairing between the $\mathrm{Li}^{+}$cations and the anions (as it was also unambiguously depicted from heteronuclear NMR correlations measurements $\left.{ }^{14,15}\right)$. Such a strong association scheme explains also the consequent decrease in the anion mobility. Throughout this manuscript we refer to the concept of ion pairing but other expressions such as Li complexation could also be used as it is relevant to many chemistry subfields.

\subsection{Ionic conductivity $\sigma$}

Fig. 3 also presents the change in ionic conductivity as Li salt is added to the $\mathrm{BMIMBF}_{4}$ ionic liquid. Again, both the experimental and simulated data are found to follow consistently the same behavior, therefore confirming that our molecular dynamics is a reasonable strategy to probe the microscopic mechanisms involved in the structure and dynamics of these complex salts. 


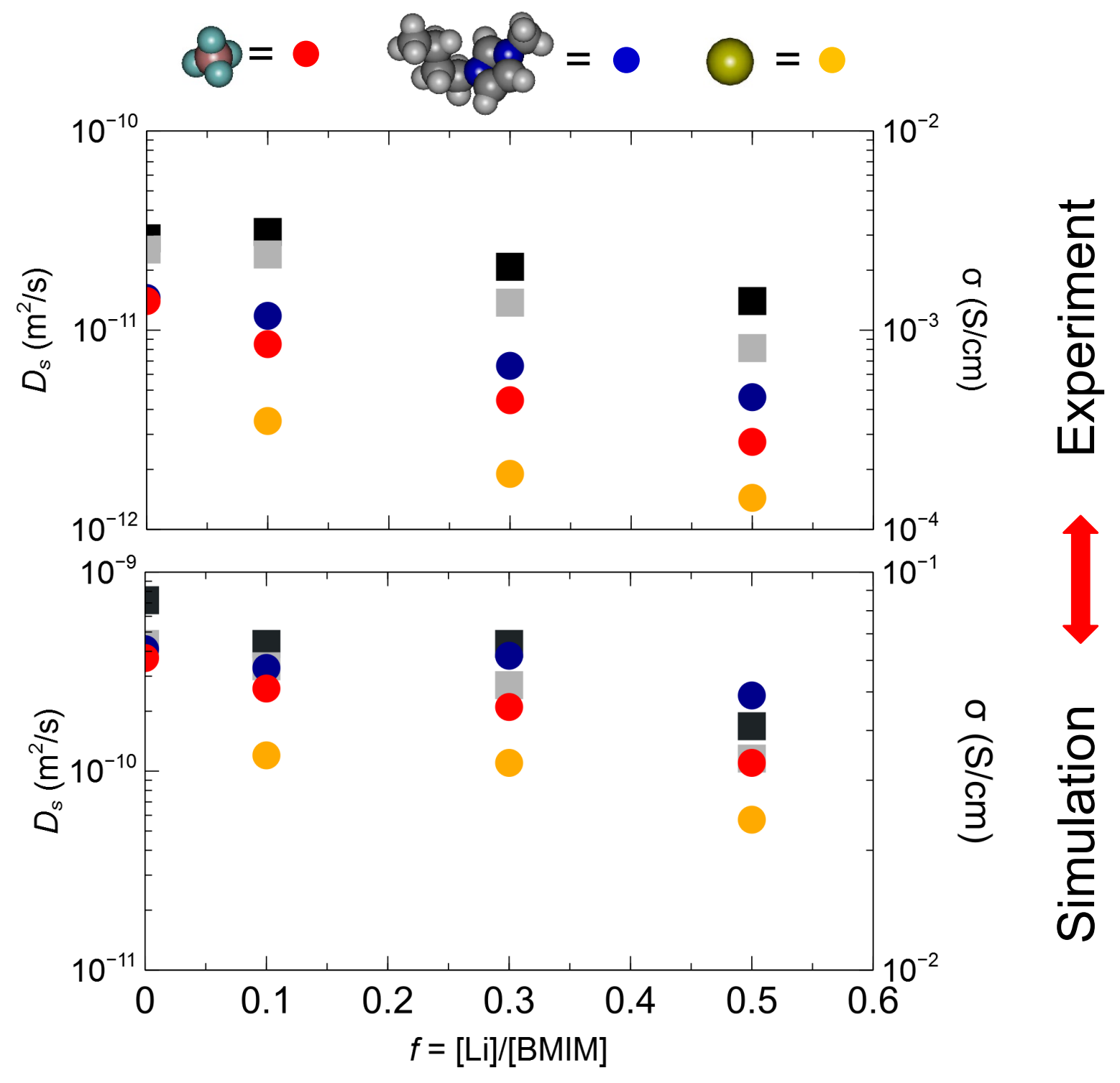

Fig. 3. Self-diffusion coefficient $D_{\mathrm{s}}$ and ionic conductivity $\sigma$ for ionic liquid-based electrolytes as a function of the cation mole fraction $f$ of $\mathrm{Li}^{+}$: (top) experimental values measured at $T=298 \mathrm{~K}$ by means of PFG-NMR and complex impedance spectroscopy and (bottom) molecular dynamics at $T=500 \mathrm{~K}$. The red, blue, and yellow circles correspond to the self-diffusifivities $D_{\mathrm{s}}$ for the $\mathrm{BF}_{4}$ anion, $\mathrm{BMIM}$ cation and $\mathrm{Li}$ cation, respectively. The grey squares denote the ionic conductivity $\sigma$ as measured experimentally and using molecular dynamics. The black squares correspond to the Nernst-Einstein conductivity predicted from the cation and anion selfdiffusivities. Error bars estimated to $\pm 10 \%$ are smaller than symbols. 
The experimental value obtained at room temperature for pure $\mathrm{BMIMBF}_{4}(\sigma=2.5 \mathrm{mS} / \mathrm{cm})$ is in fair agreement with those reported in the literature around $3.5 \mathrm{mS} / \mathrm{cm} .{ }^{61}$ Such a discrepancy may be explained by the purity of the ionic liquid (i.e., the purification process used prior to its use in experiments), the amount of water (i.e., the procedure of drying used prior to experimental measurements), or even the degradation of chemicals $\left(\mathrm{BF}_{4}\right.$ may be altered by hydrolysis, oxidation, dismutation and other ageing processes). Addition of lithium salt also leads to a significant decrease of the ionic conductivity (as was already observed for other lithium salt/ionic liquid electrolytes. ${ }^{14,31,56,60}$ This conductivity decrease is related to the strong decrease of ion mobility and the higher viscosity of solutions upon lithium addition. This will be discussed in the following sections where MD will be used to gain microscopic insights into the molecular mechanisms involved.

\section{Discussion}

\subsection{Structure}

Partial pair distribution functions $G_{i j}(r)$. The total structure factor can be calculated from MD trajectories as a weighted sum of all partial structure factors $S_{i j}(Q)$ between each pair of atoms $i, j\left(\right.$ eq. 2): ${ }^{62}$

$$
S(Q)-1=\left(\sum_{i=1}^{n} c_{i} b_{i}\right)^{-2} \sum_{i, j}^{n}\left(c_{i} c_{j}\right)^{1 / 2} b_{i} b_{j}\left[S_{i j}^{A L}(Q)-\delta_{i j}\right]
$$

In this expression, $S_{i j}^{A L}(Q)$ are derived within the Ashcroft-Langreth formalism ${ }^{63}$ from the partial radial distribution function, $G_{i j}(r)$, between each atom pair $i$ and $j$ :

$$
S_{i j}^{A L}-\delta_{i j}=4 \pi \rho\left(c_{i} c_{j}\right)^{1 / 2}=\int_{0}^{\infty} r^{2}\left[G_{i j}(r)-1\right] \frac{\sin Q r}{Q_{r}} d r
$$

where $\delta_{i j}$ are the Kronecker delta function, $c_{i}=N_{i} / N$ the mole fraction of atom type $i, b_{i}$ the scattering length of atom type $i$ (which is directly related to the number of electrons in the atom for X-ray scattering). These calculations make it possible to analyze the liquid organization from 
an atomic site-to-site perspective, but also to group/add a series of partial structure factor $S_{i j}(Q)$ to highlight the contributions and interactions of specific chemical groups or species.

Fig. 4 presents a series of the most representative pair radial distribution functions $G_{i j}(r)$ and the corresponding pair scattering functions $S_{i j}(Q)$ for different compositions $f$. For this purpose, the BMIM cation behavior was found to be well described by choosing specific sites: nitrogen atoms $\mathrm{N}$ on the ionic imidazolium moiety and the $\mathrm{C}_{\mathrm{s}}$ carbon on the alkyl chain (see Figure $\mathrm{S} 1$ in the Supporting Information for atom labelling). While the two $\mathrm{N}$ atoms in the imidazolium cation are chemically different (partial charges and local geometry), they possess identical or nearly identical scattering cross-sections. As a result, in terms of neutron or X-ray scattering, such $\mathrm{N}$ atoms contribute similarly to the structure factor $S(Q)$ and, hence, to the radial distribution functions $G(r)$ (since the latter is related to the former through inverse Fourier transformation). As a result, while these two $\mathrm{N}$ atoms possess slightly different electrostatic charges, they contribute to the same extent to the features of the total and partial structure factors. In particular, in our detailed analysis of the origin of the prepeak in the structure factor, splitting the partial structure factors involving the $\mathrm{N}$ atoms into the two subpopulations would lead to the same picture. On the other hand, the anion can be simply described using the central B atom. The first peak in the different $G_{i j}(r)$ functions allows one to get microscopic information on the nearest neighbor distribution (i.e. coordination shell) while oscillations at larger distances $r$ correspond to the mid-to-long range correlations (which are often more easily visualized in the $G_{i j}(r)$ functions than in the $S_{i j}(Q)$ functions). Even if the relative contribution of some of these partial scattering functions in the total scattering function $S(Q)$ may be negligible (due to the small concentration $c_{i}$ of the atoms and/or the intrinsic values of the scattering lengths $b_{i}$ ), the features in these curves provide key information on the structural correlation at the atomic scale. In the pure ionic liquid, oscillations at distances up to $40 \AA$ are observed in the $G_{i j}(r)$ curves, therefore providing evidence for strong structural organization inside these ionic liquids (this long range ordering is $3-5$ times the characteristic ion size). This is also reflected by the narrow linewidth 
of the different $S_{i j}(\mathrm{Q})$ correlation peaks which are located in the two domains $0.8-1.1(\mathrm{~N}-\mathrm{B}, \mathrm{B}-\mathrm{B}$ and $\mathrm{C}_{\mathrm{s}}-\mathrm{B}, \mathrm{N}-\mathrm{N}$, Fig. 3a, 3b, 3c and S3 respectively) and $1.3-1.6 \AA^{-1}\left(\mathrm{C}_{\mathrm{s}}-\mathrm{B}\right.$, Fig. 3c).

Upon addition of lithium cations, the organization of the imidazolium ion is affected in a nonuniform fashion: structural correlations related to the ionic imidazolium core become broader (NB - Fig 4.a, and N-N presented in supplementary informations - Fig S3.a and S3.b) while those involving the aliphatic chain display an additional feature at lower $Q\left(\mathrm{Q}<0.5 \AA^{-1}\right)$. A remarkable trade-off between two structural schemes is observed in the cation tail-anion contribution (isobestic point in the partial scattering contribution $\mathrm{S}_{\mathrm{CS}-\mathrm{B}}$, evidencing an equilibrium between two coordination schemes as observed for other systems ${ }^{64}$ ). The structural correlations between $\mathrm{Li}^{+}$and the two moieties of the imidazolium also differ in the antipeaks observed at two different scattering vectors: $0.8 \AA^{-1}$ for the cation head (N-Li correlations, Fig S4.a and S4.b) and $0.4 \AA^{-1}$ for the cation tail locations $\left(\mathrm{C}_{\mathrm{s}}\right.$-Li correlations, Fig S4.c and S4.d), respectively). Such features also indicate a long range structuration of the $\mathrm{Li} / \mathrm{IL}$ salt. However, upon addition of $\mathrm{Li}^{+}$, as expected, the major structural modifications are measured around the $\mathrm{BF}_{4}^{-}$anion with the $G_{\mathrm{B}-\mathrm{B}}(r)$ functions highlighting significant modification of the anion subnetwork. In the pure ionic liquid, a correlation length of $\sim 7.5 \AA$ is measured while an additional structural network characterized by a B-B distance of $\sim 5.1 \AA$ appears as soon as $\mathrm{Li}^{+}$ions are added. 

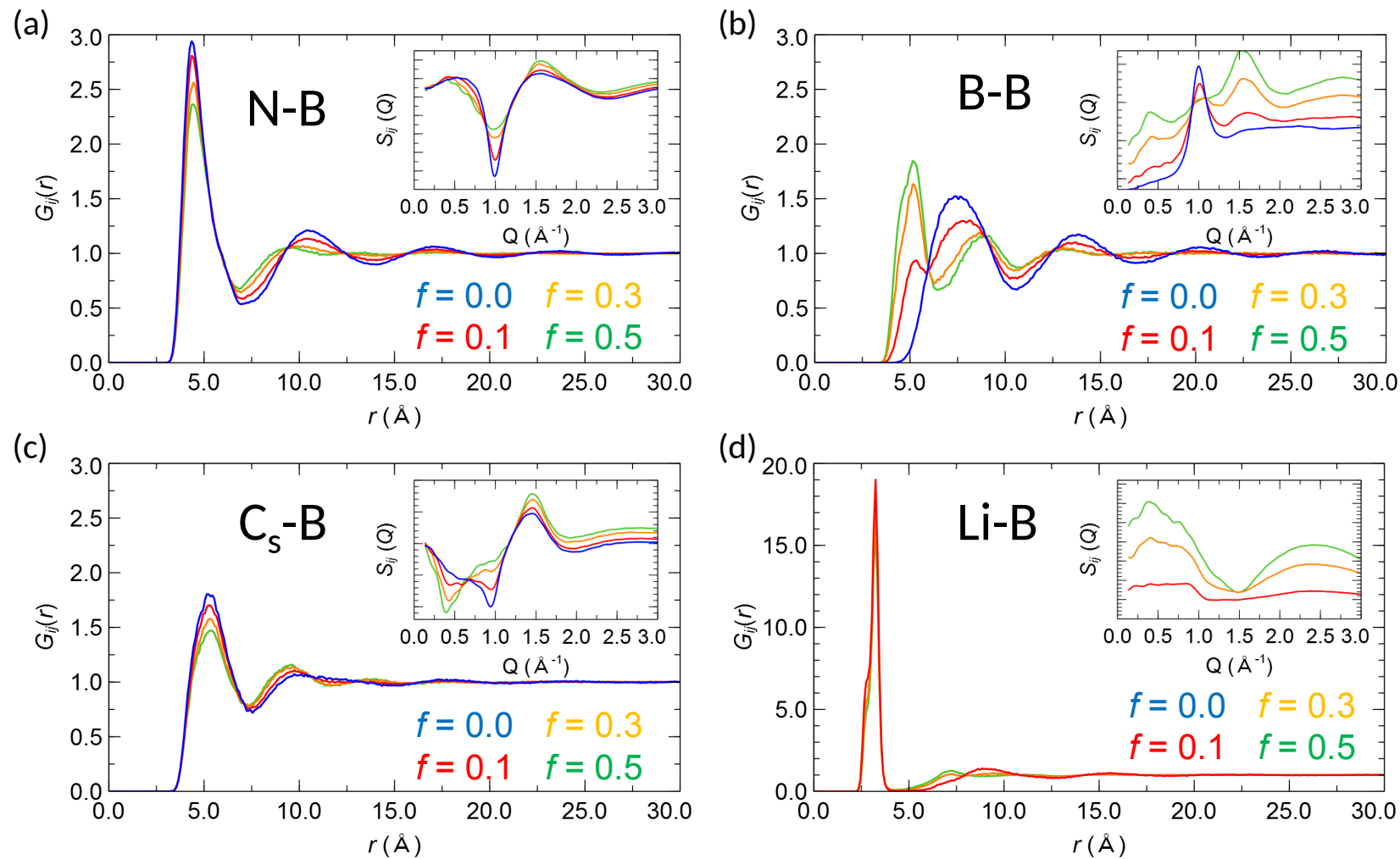

Fig. 4. Partial radial distribution functions $G_{i j}(r)$ for an ionic liquid-based electrolyte BMIM/BF containing a cation mole fraction $f$ of lithium cations between: (a) $\mathrm{N}$ atom in BMIM with B atom in $\mathrm{BF}_{4}$, (b) $\mathrm{B}$ atoms in $\mathrm{BF}_{4}$, (c) $\mathrm{C}_{5}$ atom in $\mathrm{BMIM}$ with $\mathrm{B}$ atom in $\mathrm{BF}_{4}$, and (d) $\mathrm{Li}$ ions and $\mathrm{B}$ atoms in $\mathrm{BF}_{4}$. As indicated in the graph, the data are shown for four different mole fractions $f=$ $0.0,0.1,0.3$ and 0.5 . For each partial radial distribution function, the corresponding structure factor $S_{i j}(Q)$ is shown in the insert.

Concomitantly, the development of a coordination structure ' $\mathrm{Li}$...BF $\mathrm{BF}_{4}$ is observed with $\mathrm{Li}^{-\mathrm{BF}_{4}}$ (Figure 4.d) and Li-Li correlation distances of $3.2 \AA$ and $4.5 \AA$, respectively (Figure S5.a and S5.b). These two distances are consistent with a coordination shell of $\mathrm{BF}_{4}^{-}$around the alkali cations and the formation of ${ }^{\prime} \mathrm{Li}\left(\mathrm{BF}_{4}\right)_{\mathrm{n}}{ }^{(\mathrm{n}-1)-1}$ clusters ${ }^{65}$ inside the $\mathrm{BMIM}-\mathrm{BF}_{4}$ IL matrix. The average number of the different neighbors around $\mathrm{Li}^{+}$ions, which can be estimated by integrating the $G_{i j}(r)$ functions between the ion centers of mass, are summarized in Table 1 (see also Figure S6 in the Supporting Information where we show the integrated functions). These values suggest 
that the $\mathrm{Li}^{+}$ions are surrounded by about $4 \mathrm{BF}_{4}^{-}$anions in their coordination shell. Moreover, the presence of $\mathrm{Li}^{+}$second neighbors around $\mathrm{Li}^{+}$cations, which is already non negligible even at low concentration, increases in a monotonous fashion with the alkali content.

Table 1. $\mathrm{Li}^{+}$coordination and molecular environment in an ionic liquid-based electrolyte $\mathrm{LiBF}_{4} / \mathrm{BMIMBF}_{4}$ as a function of the $\mathrm{Li}$ mole fraction $\mathrm{f}$. The values $(*)$ and $(* *)$ were determined by integrating the corresponding $G(r)$ as shown in the Supporting Information.

\begin{tabular}{llll}
\hline \hline Property & $f=0.1$ & $f=0.3$ & $f=0.5$ \\
\hline number of $\mathrm{BF}_{4}^{-}$ion around $\mathrm{Li}^{+(*)}$ & 3.9 & 4.0 & 4.1 \\
Number of $\mathrm{Li}^{+} \leq 7 \AA{ }^{(* *)}$ & 0.6 & 1.8 & 2.8 \\
Mean Li-Li statistical distance & 15.4 & 10.7 & 9.2 \\
$\% \mathrm{Li}^{+}$inside clusters $\left(\mathrm{d}_{\mathrm{Li}}-\mathrm{d}_{\mathrm{Li}} \leq 7 \AA\right)$ & 21 & 53 & 78 \\
\hline
\end{tabular}

Structure factors. Fig. 5 shows the total structure factor $S(Q)$ and its partial contributions $S_{i j}(Q)$ between ion centers of mass (the different cation/cation, anion/anion, cation/anion contributions are shown). Both the data for the pure ionic liquid and the ionic liquid containing $\mathrm{Li}^{+}$ions are considered. The structure factor for $\mathrm{BMIMBF}_{4}$ presents a broad peak centered at about $Q \sim 1.4-$ $1.5 \AA^{-1}$ and a small and broad shoulder around $Q \sim 0.6 \AA^{-1}$. In contrast, the different ion/ion contributions present sharp correlation peaks around $Q \sim 1 \AA^{-1}$. Like with other ILs,,$^{54,66}$ the cation/cation and anion/anion partial structure factors display a positive peak which is counterbalanced by a negative peak - also called anti-peak - in the anion-cation correlations. These features, which are rather narrow, nearly perfectly compensate each other when they are summed. This reflects the high degree of organization in these salts which involves a well ordered sequence of alternating positive and negative charges. As a result of this compensation, 
the total structure factor displays mostly a single scattering peak at about $Q \sim 1.4 \AA^{-1}$ which corresponds to cation/cation and cation/anion correlations (while the shoulder at $Q \sim 0.6 \AA^{-1}$ arises from anion-anion correlations).

The decomposition of the structure factor for $\mathrm{BMIMBF}_{4} / \mathrm{LiBF}_{4}$ with $f=0.5$ into partial ionic contributions allows identifying the significant structural changes upon $\mathrm{Li}^{+}$salt addition. Due to the small Li mole concentration and small molecular mass/X-ray scattering length, all contributions arising from correlations with $\mathrm{Li}$ ions contribute only little to the total stucture factor $S(Q)$. Therefore, like for pure IL, the prevailing partial structure factors are those for the cation/cation, anion/anion (peaks) and cation/anion (anti-peak) at about $Q \sim 1 \AA^{-1}$. These peaks are broader in the Li-IL electrolyte than in pure IL, which provides evidence for a lower degree of organization in the mixed system. However, like for the pure ionic liquid, when these contributions are summed, they vanish as a consequence of alternating charge sequence. The feature at $Q \sim 1.4 \AA^{-1}$ is still a combination of positive contributions of these different ion pairs. In fact, the most significant modification appearing upon $\mathrm{Li}^{+}$addition is observed in the low $Q$ range for the anion/anion and anion/cation contributions (which display peak and anti-peak features, respectively). Because these peaks/anti-peaks have different amplitudes, a resulting peak is observed at $Q \sim 0.4-0.5 \AA^{-1}$. This indicates that $\mathrm{Li}^{+}$addition to the IL leads to a second series of alternating charges between $\mathrm{BMIM}$ and $\mathrm{BF}_{4}$ ions with a larger coherence length. Moreover, the increase in the anion/anion correlations as seen in the total $S(Q)$ upon adding $\mathrm{Li}^{+}$ suggests a reorganization of the $\mathrm{BF}_{4}^{-}$anions with the formation of more structured ionic clusters involving $\mathrm{BF}_{4}^{-}$. Experimentally, the change of $\mathrm{BF}_{4}^{-}$coordination sphere is also depicted from the variations of ${ }^{7} \mathrm{Li}$ and ${ }^{19} \mathrm{~F}$ NMR chemical shifts (Fig. S7). 

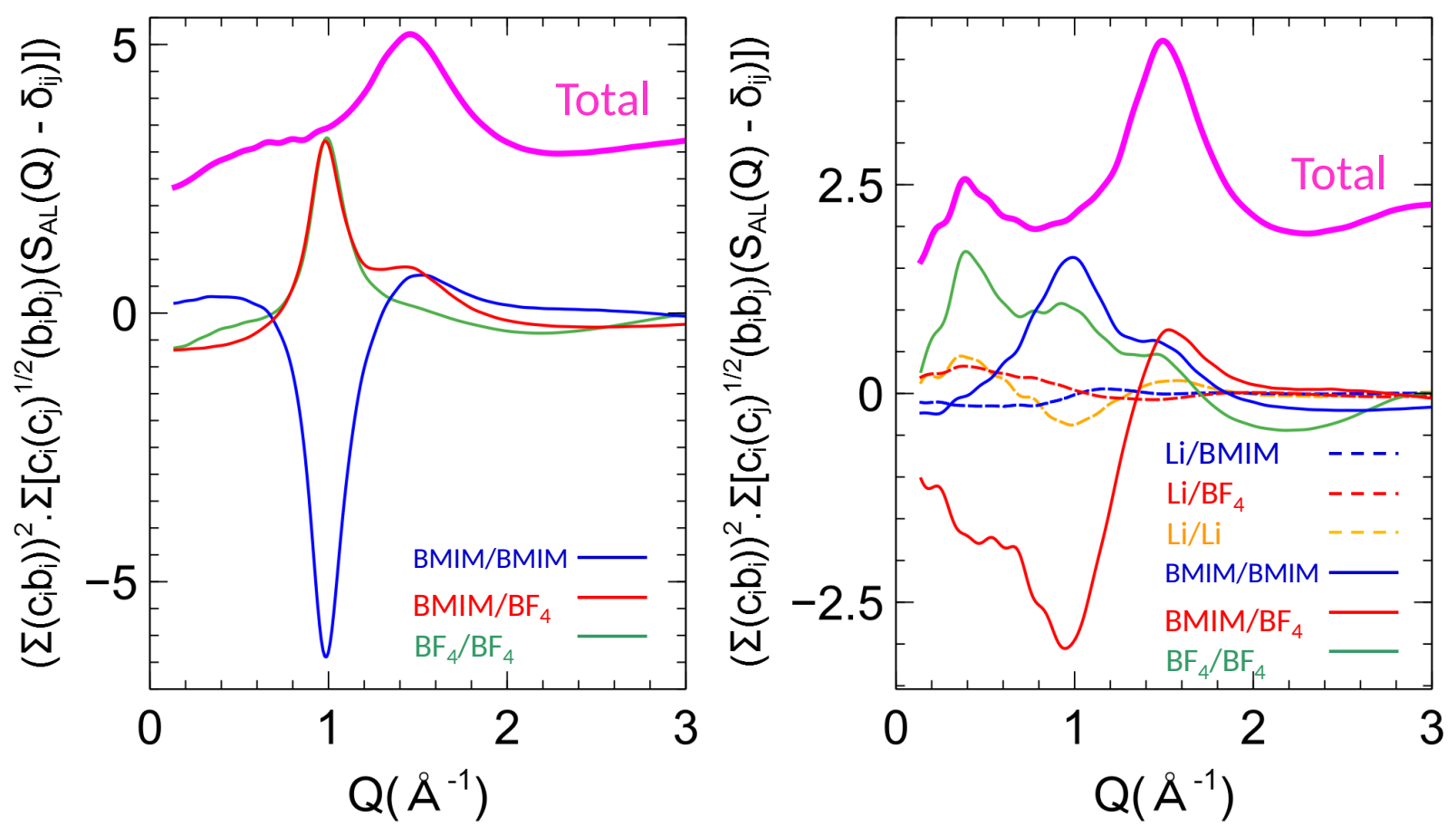

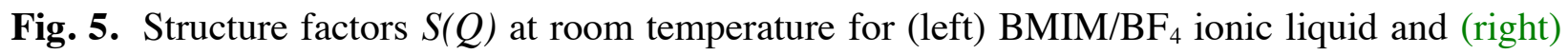
for the same ionic liquid containing a Li cation fraction $f=0.5$. The magenta data show the total structure factor while the different contributions are shown in green, blue, and red as indicated in the graph. Note that the total structure factor curve has been shifted up for the sake of clarity. For the electrolyte containing $\mathrm{Li}$, the dashed lines show the contributions involving Li ions. The LiLi contribution has been multiplied by $\times 100$ for visualization purpose as the Li scattering length and concentration are too small to significantly contribute to the total scattering function.

Molecular configurations. The structural modifications observed in the partial and total structure factors can be pictured from typical molecular configurations generated along the MD simulations. Fig. 6 shows typical cluster aggregations observed for the ionic liquid highly doped with $\mathrm{Li}^{+}(f=0.5)$. As expected, the main aggregation structure corresponds to isolated $\mathrm{Li}$ or $\mathrm{Li}$ dimers for the low alkali concentration while larger aggregates are observed upon increasing $f$. More in detail, in an isolated $\mathrm{Li}^{+}$cation configuration, the $\mathrm{Li}^{+}$is coordinated by $4 \mathrm{BF}_{4}^{-}$forming a tetrahedral geometry. In some of these $\left[\mathrm{Li}\left(\mathrm{BF}_{4}\right)_{4}\right]^{3-}$ units, one of the $\mathrm{BF}_{4}$ anions may show a 
bidentate association - the preferred coordination state being either 4 or $5 .{ }^{67}$ Mono and bidentate forms also manifest themselves in the center of mass $G(r)$ where a shoulder is observed on the left side - i.e. smaller distances - of the $\mathrm{Li}^{-\mathrm{BF}_{4}}$ correlation peak (Figure 4). As expected, most of the $\mathrm{BF}_{4}^{-}$anions are monodentate with only slight variation upon varying the ratio $f$ (with monodentate configuration being favored). A typical Li dimer structure is illustrated in Fig. 6 here, the two $\mathrm{Li}^{+}$are coordinated by two bridging $\mathrm{BF}_{4}^{-}$ions and the coordinating cation is completed by a monodentate terminal $\mathrm{BF}_{4}{ }^{-}$. Some of the dimers are also connected by only one bridging $\mathrm{BF}_{4}^{-}$. These various configurations are also encountered in the bigger clusters which develop with increasing $f$ as shown in the other molecular configurations in Fig. 6. The low density of the large clusters is noticeable with such geometrical features leading to interesting dissolution versus precipitation effects. The dissolution of a single $\mathrm{Li}^{+}$requires $4 \mathrm{BF}_{4}{ }^{-}$to achieve full coordination while only one $\mathrm{BF}_{4}^{-}$is necessary to complete charge stoichiometry; this leads to favorable formation of large and multicharged anion $\mathrm{Li}\left[\left(\mathrm{BF}_{4}\right)_{4}\right]^{3-}$. In Li dimers, six or seven $\mathrm{BF}_{4}^{-}$ take part to form a stable aggregated structure depending whether the two lithium ions are bridged by a double or a single $\mathrm{BF}_{4}^{-}$anion (leading to multicharged anions $\operatorname{Li}_{2}\left[\left(\mathrm{BF}_{4}\right)_{6}\right]^{4-}$ and $\mathrm{Li}_{2}\left[\left(\mathrm{BF}_{4}\right)_{7}\right]^{5-}$, respectively). Moreover, when larger aggregates develop such as those shown in Fig. 6, a negatively multicharged anion of low density grows. Such ratio of negative charges fixed in a specific location leads to a specific geometry of charge distribution which severely constrains the location of the $\mathrm{BMIM}^{+}$for charge neutralization. Indeed, positive imidazolium cores cannot get closer to the negative charges because of steric constraints - which could explain the decrease in head-head and head-anion organization while tail-tail configurations become more ordered. 
(a)

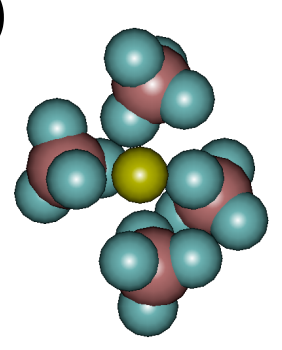

(b)

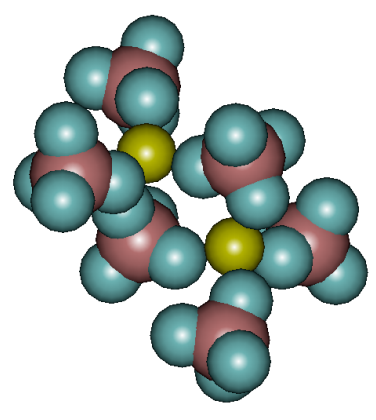

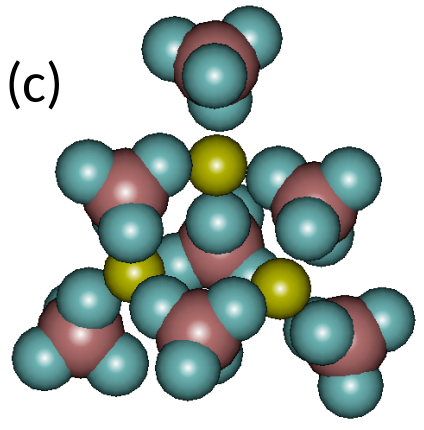

(d)

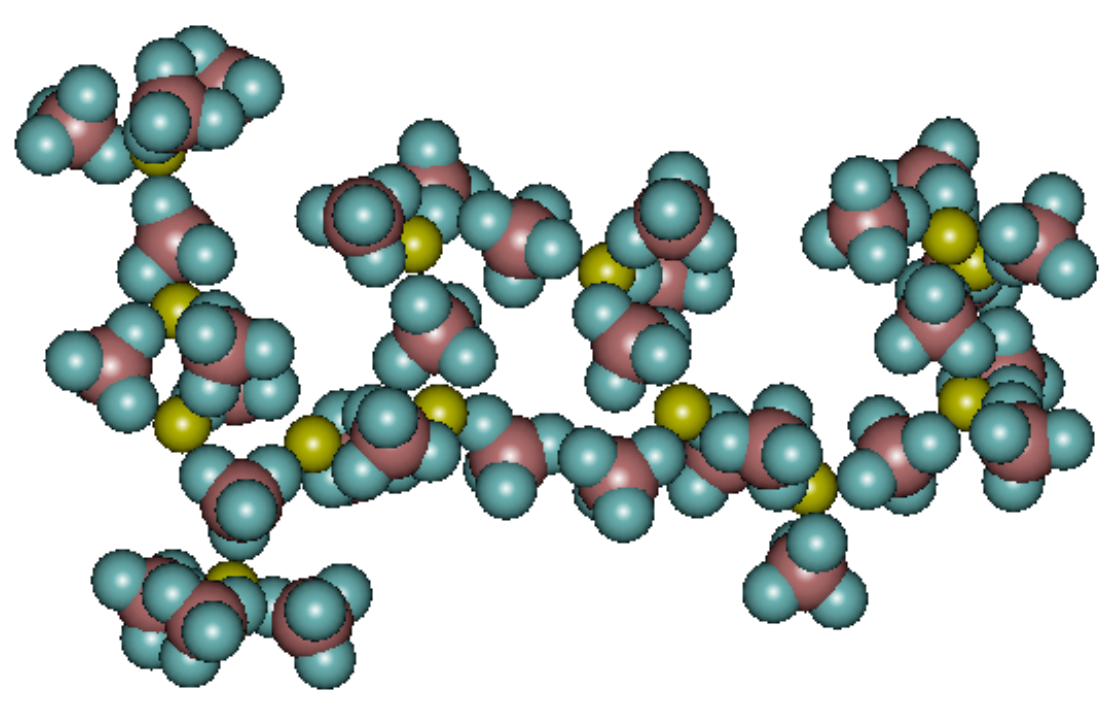

Fig. 6. Typical molecular clusters as identified in molecular dynamics simulations of a $\mathrm{BMIM} / \mathrm{BF}_{4}$ ionic liquid containing a Li cation fraction $f=0.5$. The yellow spheres are Li cation while the purple and cyan spheres are $\mathrm{B}$ and $\mathrm{F}$ atoms in $\mathrm{BF}_{4}$ ions (the $\mathrm{BMIM}$ ions are not shown for the sake of clarity). The molecular configurations in (a), (b), (c), and (d) display a monomer, dimer; trimer, and 13-mer, respectively.

\subsection{Dynamics}

Mean square displacements and self-diffusivities. Fig. 7 presents individual mean square displacements (MSD) computed at $T=500 \mathrm{~K}$ for various sites $i$ of the BMIM and Li cations and $\mathrm{BF}_{4}$ anions: $\Delta r_{i}^{2}(t)=\left\langle\left(r_{i}(t)-r_{i}(0)\right)^{2}\right\rangle$ (in addition to the different atomic sites $i$, the center of mass of these ionic species is also considered). In this formula, the brackets denote statistical average over representative configurations of the system in the NVT ensemble. Such trajectories 
allow us to decipher various motional modes. (a) At shorter time scales ( $1 \mathrm{fs}-100 \mathrm{fs}$ ), individual MSD involve motions in the picometer range which correspond to Debye Waller and localized vibration modes. This domain therefore provides information that can be compared to broad band dielectric, Raman, and infrared spectroscopies. (b) For time scales longer than 5 ns, displacements are in the nanometer range, i.e. much larger than the hydrodynamic ion radius, so that all atoms display the same MSD (because individual displacements are merged into the same ion motion mode). This regime will be specifically studied in the next section to estimate the different ion self-diffusivities and ionic conductivity. (c) In the intermediate regime, the MSD for the different atoms differ from each other as the underlying motions are in the $\sim 0.1$ nanometer range and correspond to localized atomic fragments such as rotation, translation and/or chain conformation rearrangement. As an example, the MSD corresponding to the methyl group at the end of the butyl chain $\left(\mathrm{H}_{\mathrm{but}}\right)$ is larger than that of the methyl linked to the imidazolium core $\left(\mathrm{H}_{\text {met }}\right)$. Experimentally, this regime is usually probed by means of Quasi-Elastic Neutron Scattering and Neutron Spin Echo ${ }^{49,68,69,70,71,72}$, Nuclear Magnetic Resonance (NMR) through relaxation or dipolar coupling measurements ${ }^{14,15,73}$ and from local rotational diffusion of fluorescent probes..$^{74}$

The self-diffusion coefficients $D_{\mathrm{s}}$ for the different ions can be obtained readily from the MSD using (1) the velocity autocorrelation method or (2) Einstein formula in the Fickian regime: $\Delta r^{2}(t) \sim 6 D_{s} t$. However, in practice, the former method was found to lead to poorly converged values because of the limited statistics and the heterogeneous structure of ionic liquid electrolytes. In contrast, the latter method is more robust but requires to compute trajectories on a large time-scale. Fig. 7 shows that, even if a temperature of $500 \mathrm{~K}$ is considered in the molecular dynamics simulation, the Fickian regime is not reached before $t \sim 10^{3} \mathrm{ps}$. In additon to plotting MSD versus $t$ in a $\log$-log scale, it is instructive to plot the $\log$ derivative $\partial \log \left(\Delta r^{2}(t)\right) / \partial \log t$ to check the time dependence of $\Delta r^{2}(t)$. As shown in Fig. 7, three regimes are clearly identified when considering time scales spanning from $10^{-2}$ to $10^{4} \mathrm{ps}$. Up to $\sim 0.1 \mathrm{ps}$, the system is in the 
ballistic regime $\Delta r^{2} \sim t^{2}$ while the Fickian i.e. diffusive regime $\Delta r^{2} \sim t$ is obtained for $t>1 \mathrm{~ns}$. In between these two asymptotic limits, $\Delta r^{2} \sim t^{\alpha}$ with large, continuous variations between $\alpha=2$, 0.5 and then 1 in the time range $1-10$ ps. This large temporal domain corresponds to the crossover between molecular mobility mechanisms and nanoscale diffusion. ${ }^{75}$ As shown in Fig. 7, the behavior of $\mathrm{BMIM}^{+}, \mathrm{BF}_{4}{ }^{-}$and $\mathrm{Li}^{+}$is rather similar to that observed for the pure ionic liquid with the exception of the short time regime for the alkali ion $\mathrm{Li}^{+}$which displays some oscillations. This behavior, which is clearly seen in the log derivative, corresponds to wobbling and rattling effect inside the Li coordination shell ${ }^{58,60,76,77}$ corresponding to far-IR spectroscopic modes. ${ }^{78}$ Another important difference between the Li-free ionic liquid and its counterpart containing $\mathrm{Li}$ lies in the fact that the Fickian regime for the $\mathrm{BF}_{4}^{-}$and $\mathrm{Li}^{+}$ions in the salt mixture is reached at much longer times (> $5 \mathrm{~ns}$ ). The $\mathrm{MSD}$ for the $\mathrm{BMIM}^{+}$and $\mathrm{BF}_{4}^{-}$nearly overlap in the Fickian regime for pure $\mathrm{BMIMBF}_{4}$ while they differ when $\mathrm{Li}^{+}$is added. In the latter situation, the MSD for $\mathrm{BF}_{4}^{-}$is lying between those for $\mathrm{BMIM}^{+}$and $\mathrm{Li}^{+}$. This reflects the strength of association/ion-pairing between anions and the alkali cations.

The ion self-diffusivities obtained by means of MD are compared in Table 2 with their experimental counterparts obtained using NMR measurements at $298 \mathrm{~K}$. As discussed above, due to methodological constraints (trajectory duration to reach Fickian regime), the MD results were obtained at $T=500 \mathrm{~K}$ so that comparison against experimental results is only qualitative. Yet, as shown in Table 2, the change observed in the simulated ion self-diffusivities upon increasing the $\mathrm{Li}$ content is consistent with the experimental results. First, in the absence of $\mathrm{Li}^{+}, D_{\mathrm{s}}^{\mathrm{BMIM}} \sim D_{\mathrm{s}}^{\mathrm{BF} 4}$; this can be rationalized by the strong correlations between individual displacements in such molten salts which lead to unified motion. Second, upon adding $\mathrm{Li}^{+}$, the ion self-diffusivities decrease and become different from each other; $D_{\mathrm{s}}{ }^{\mathrm{BMIM}}>D_{\mathrm{s}}{ }^{\mathrm{BF} 4}>D_{\mathrm{s}}{ }^{\mathrm{Li}}$. The diffusion coefficient for all ions decreases as $\mathrm{Li}^{+}$content increases. This effect is less pronounced in the simulated data than in the experimental data; this is thought to be due to the larger temperature used in the 
MD approach. The different ion self-diffusivities can be used to estimate the transference number $t_{\mathrm{Li}}$ for $\mathrm{Li}^{+}$- a key quantity in electrochemistry as it is directly linked to process efficiency:

$$
t_{L i}=\frac{N_{L i} D_{s}^{L i}}{N_{L i} D_{s}^{L i}+N_{B M I M} D_{s}^{B M I M}+N_{B F_{4}} D_{s}^{B F_{4}}}
$$

where $N_{L i}, N_{B M I M}$ and $N_{B F 4}$ are respectively the molar fraction of Li, BMIM and $\mathrm{BF}_{4}$ in the electrolyte.
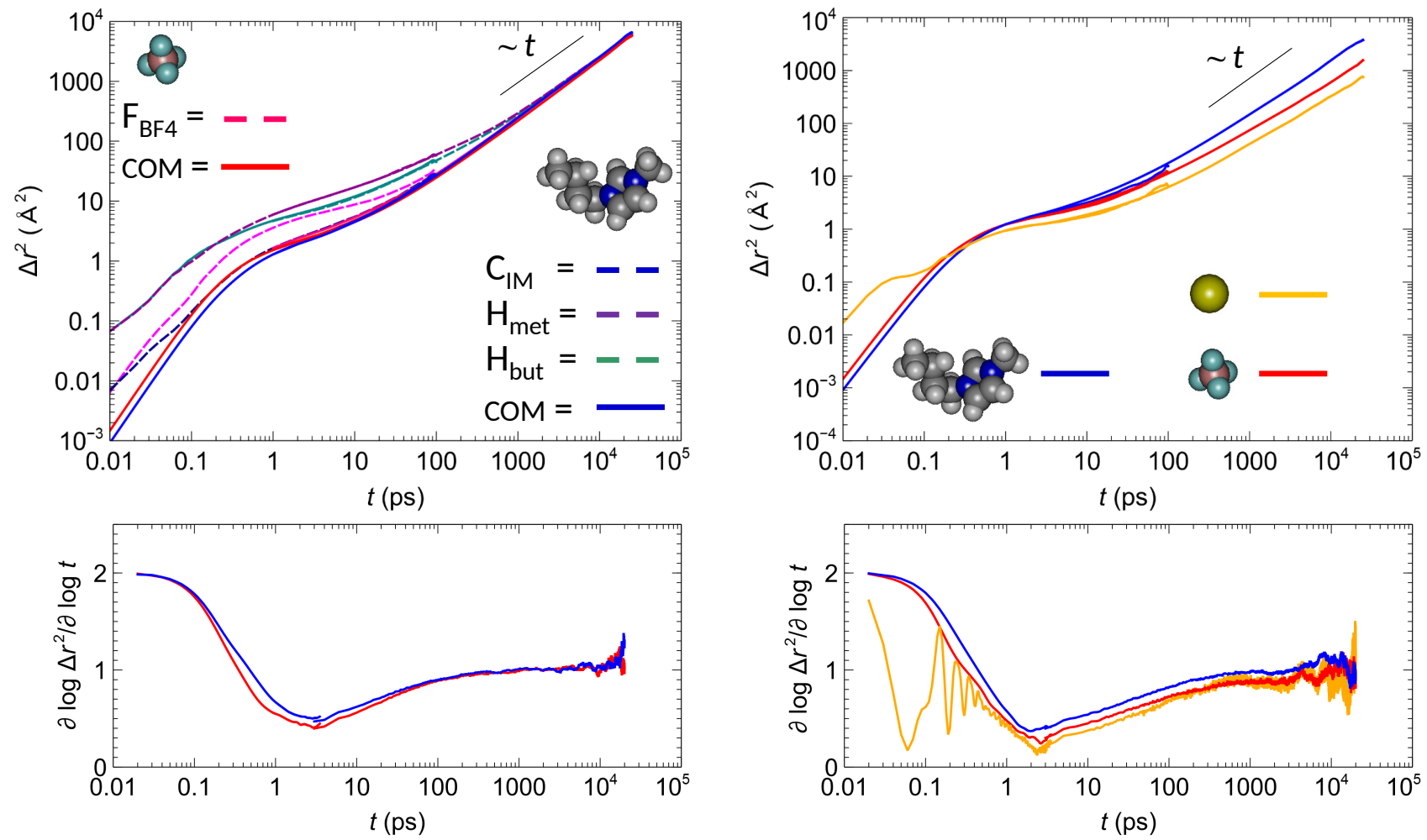

Fig. 7. (left) Mean square displacements $\Delta r^{2}$ as a function of time $t$ for $B M I M / B F_{4}$ ionic liquid at $T=500 \mathrm{~K}$. The red and blue solid lines denote the results for the center of mass (COM) of the $\mathrm{BF}_{4}$ anion and BMIM cation, respectively. As indicated in the graph, the dashed lines correspond to the mean square displacements for different atomic sites in the cation and anion. (right) Same as left but for the $\mathrm{BMIM} / \mathrm{BF}_{4}$ ionic liquid containing a Li cation fraction $f=0.5$. The red, blue and yellow solid lines correspond to the results for the center of mass $(\mathrm{COM})$ of the $\mathrm{BF}_{4}$ anion, BMIM cation, and Li cation. In both panels, the black solid segment indicates the expected 
fickian regime $\Delta r^{2} \sim t$. The plot in the bottom indicates the exponent $\alpha$ as a function of time $t$ estimated as $\Delta r^{2} \sim t^{\alpha}$. The oscillations in the exponent $\alpha$ for $\mathrm{Li}$ arises from rattling within molecular cages (see text) (same color/symbol code as in the main figures). Short and long trajectories (see computational methods section) are merged to probe dynamics on a large time scale.

Table 2. Transport properties of ionic liquid-based electrolytes $\mathrm{LiBF}_{4} / \mathrm{BMIMBF}$ as a function of the Li cation fraction $f$. Both experimental and simulated data are reported for the selfdiffusion coefficients of the different species $\left(\mathrm{BMIM}, \mathrm{BF}_{4}\right.$ and $\left.\mathrm{Li}\right)$ and the lithium transference $t_{L i}$. For each quantity, the technique and temperature used are indicated. While the transference is a dimensionless number, the self-diffusivity $D_{\mathrm{s}}$ is given in units $10^{-12} \mathrm{~m}^{2} / \mathrm{s}$ (experimental errors on $D_{i}$ determined from NMR and MSD are $\left.\pm 10 \%\right)$.

\begin{tabular}{|c|c|c|c|c|c|c|c|c|c|c|c|}
\hline \multirow[t]{2}{*}{ Property } & \multicolumn{2}{|l|}{$f=0$} & \multicolumn{3}{|c|}{$f=0.1$} & \multicolumn{3}{|c|}{$f=0.3$} & \multicolumn{3}{|c|}{$f=0.5$} \\
\hline & BMIM & $\mathrm{BF}_{4}$ & $\mathrm{Li}$ & BMIM & $\mathrm{BF}_{4}$ & $\mathrm{Li}$ & BMIM & $\mathrm{BF}_{4}$ & $\mathrm{Li}$ & BMIM & $\mathrm{BF}_{4}$ \\
\hline $\mathrm{D}_{\mathrm{s}}^{\mathrm{NMR}}(298 \mathrm{~K})$ & 14.5 & 14.0 & 3.5 & 11.8 & 8.51 & 1.9 & 6.58 & 4.45 & 1.4 & 4.61 & 2.7 \\
\hline $\mathrm{D}_{\mathrm{s}}^{\mathrm{MSD}}(500 \mathrm{~K})$ & 410 & 370 & 120 & 330 & 260 & 110 & 300 & 210 & 57 & 240 & 110 \\
\hline$t_{L i}{ }^{\mathrm{NMR}}(298 \mathrm{~K})$ & $\mathrm{n} / \mathrm{a}$ & & 0.02 & & & 0.04 & & & 0.08 & & \\
\hline $\mathrm{t}_{\mathrm{Li}}{ }^{\mathrm{MSD}}(500 \mathrm{~K})$ & $\mathrm{n} / \mathrm{a}$ & & 0.02 & & & 0.05 & & & 0.10 & & \\
\hline
\end{tabular}

Such dimensionless transport numbers can be used to compare experimental and simulated data in terms of ionic transport efficiency. As shown in Table 2, the molecular simulation and experimental approaches lead to very consistent qualitative trends. In particular, both approaches show very low $\mathrm{Li}^{+}$transference numbers, which arise from the small Li self-diffusivity $D_{\mathrm{s}}^{\mathrm{Li}}$. On the other hand, despite the decrease in $D_{\mathrm{s}}^{\mathrm{Li}}$ upon increasing $f$, the transference number $t_{L i}$ increases with $f$ as a consequence of the increasing number of $\mathrm{Li}^{+}$carriers. However, quantitatively, we note that the change in $D_{\mathrm{s}}^{\mathrm{Li}}$ upon increasing $f$ differs between the simulated 
and experimental data. Experimentally, $D_{\mathrm{s}}{ }^{\mathrm{Li}}$ decreases faster with increasing $\mathrm{f}$ in the low fraction range than in the high fraction range. In contrast, with the simulated data, $D_{\mathrm{s}}^{\mathrm{Li}}$ is nearly constant for $f=0.1-0.3$ and then decreases faster upon further increasing $f$. Such discrepancies between the experimental and molecular simulation data are believed to arise from the selected force field which is not perfectly accurate in describing the effect of Li salt addition on the behavior of the ionic liquid considered here.

Conductivity and ion pairing. The ionic conductivty $\sigma$ was also assessed from MD using the two following methods: (a) the Green-Kubo formalism where $\sigma_{\mathrm{GK}}$ is obtained by integrating the electrical current autocorrelation function ${ }^{52,79}$ and (b) the Einstein formalism where $\sigma_{\mathrm{E}}$ is determined from the time dependence of the charge-weighted collective mean square displacement (the so-called collective charge transport). ${ }^{32,80}$ However, in practice, the ionic conductivity determined from the Green-Kubo approach was found to lead to poorly converged values because of the limited statistics obtained using available MD trajectories. In contrast, the ionic conductivity $\sigma_{\mathrm{E}}$ obtained using the second approach was found to lead to reasonable values (even if very long trajectories are required to reach convergence). Fig. 8 presents for both the pure ionic liquid and the ionic liquid containing a given amount of $\operatorname{Li}(f=0.5)$ the time evolution of the collective charge transport $M S D_{\text {coll }}$ of the $n$ ions contained in the electrolyte:

$$
M S D_{\text {coll }}=\sum_{i, j}^{n} z_{i} z_{j}\left\langle\left[R_{i}(t)-R_{i}(0)\right] \times\left[R_{j}(t)-R_{j}(0)\right]\right\rangle
$$

Comparing the results obtained for different simulation lengths - results not shown - showed that trajectories of at least a few $100 \mathrm{~ns}$ are required to obtain accurate data. In particular, even $50 \mathrm{~ns}$ long MD simulations were found to be insufficient to obtain very accurate values for collective quantities (such as $M S D_{\text {coll }}$ and the corresponding ionic conductivity). This illustrates the difficulty to obtain reasonable statistics due to the large uncertainty in cross-correlations (correlated ion motions). Indeed, in contrast to self-contributions, such cooperative effects have to be considered when dealing with collective phenomena such as ionic conductivity. 
The ionic conductivity $\sigma_{E}$ can be assessed from $\mathrm{MSD}_{\text {coll }}$ :

$$
\sigma_{E}=e^{2} /\left(V k_{B} T\right) . \lim _{t \rightarrow \infty} M S D_{\text {coll }}
$$

where $e$ is the electron charge, $V$ the system volume, $k_{\mathrm{B}}$ Boltzmann constant, and $T$ the temperature. As shown in Table 3, both the experimental and simulated data show that the ionic conductivity $\sigma_{\mathrm{E}}$ decreases significantly upon increasing the ratio $f$ of added $\mathrm{Li}^{+}$. Due to the use of different temperatures in the molecular simulation and experimental approaches, the ionic conductivities in Table 3 cannot be compared directly. Yet, the ionic conductivity drop observed upon increasing the $\mathrm{Li}^{+}$concentration is of comparable amplitude. Fig. 8 also shows the chargeweighted collective transport $M S D_{N E}$ if cross-correlations are neglected. ${ }^{81}$ This quantity allows defining the so-called Nernst-Einstein ionic conductivity $\sigma_{\mathrm{NE}}$ which can be assessed as : $\sigma_{N E}=e^{2} /\left(V k_{B} T\right) \lim _{t \rightarrow \infty} M S D_{N E}=e^{2} /\left(V k_{B} T\right)\left[x_{L i} D_{s}^{L i}+x_{B M I M} D_{s}^{B M I M}+x_{B F_{4}} D_{s}^{B F_{4}}\right]$

(where the second equality holds because the ion charges are unity so that $z^{2}=1$ for all ions). The dimensionless dissociation ratio, $\Lambda_{d}=\sigma_{\mathrm{E}} / \sigma_{\mathrm{NE}}$, is an important measurement of the ionicity of charge transport in a given electrolyte. 


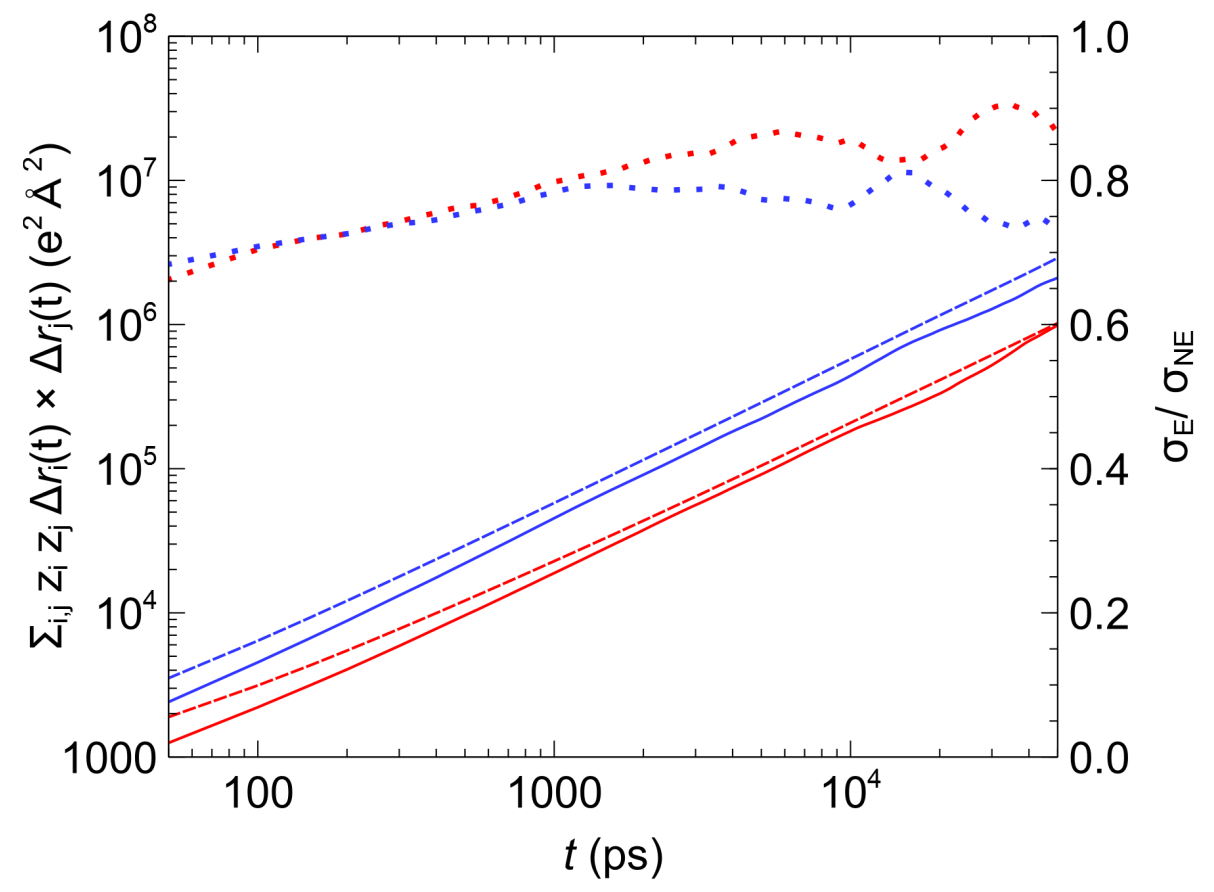

Fig. 8. Charge normalized correlations in ionic displacements as a function of time $t$ for $\mathrm{BMIM} / \mathrm{BF}_{4}$ ionic liquid (blue data) and for the same ionic liquid containing a Li cation fraction $f$ $=0.5$ (red data). The solid lines correspond to direct calculations while the dashed lines correspond to the prediction assuming an ideal conductivity where cross-correlations between different ions are neglected. For each system, the dotted line corresponds to the ratio of the simulated Einstein $\sigma_{\mathrm{E}}$ ionic conductivities and Nernst-Einstein $\sigma_{\mathrm{NE}}$ (right axis).

Fig. 8 presents $\Lambda_{\mathrm{d}}=\sigma_{\mathrm{E}} / \sigma_{\mathrm{NE}}$ as a function of time $t$. Despite a large error bar, $\Lambda_{\mathrm{d}}=\sigma_{\mathrm{E}} / \sigma_{\mathrm{NE}}$ can be estimated as $0.85 \pm 0.1$ for the pure ionic liquid and the ionic liquid-based electrolyte containing lithium. As expected, these values are lower than 1 as the Nernst-Einstein conductivity is an upper bound since it assumes no correlation between the anion/cation displacements. However, if the computed value is rather similar to those measured experimentally (see Fig. 3), the discrepancy between the experimental and simulated values increases upon increasing the lithium amount. Indeed, a noticeable decrease in $\Lambda_{d}$ is observed experimentally, indicating that 
more lithium leads to fewer charge carriers as a consequence of the increasing density of Li aggregates. ${ }^{82}$

Table 3. Charge transport properties of ionic liquid-based electrolyte $\mathrm{LiBF}_{4} / \mathrm{BMIMBF} 4$ as a function of the Li mole fraction $f$. The experimental ionic conductivity and Nernst-Einstein conductivity at $298 \mathrm{~K}$ are compared with their simulated counterpart at $500 \mathrm{~K}$ (all ionic conductivities $\sigma$ are given in units $10^{2} \mathrm{~S} / \mathrm{cm}$ ). The dissociation ratio $\Lambda_{d}$ is also reported.

\begin{tabular}{lcccc}
\hline Property & $f=0$ & $f=0.1$ & $f=0.3$ & $f=0.5$ \\
\hline$\sigma^{\mathrm{EIS}}(298 \mathrm{~K})$ & 0.254 & 0.240 & 0.137 & 0.0813 \\
$\sigma_{\mathrm{NE}}^{\mathrm{NMR}}(298 \mathrm{~K})$ & 0.289 & 0.381 & 0.208 & 0.140 \\
$\Lambda_{\mathrm{d}}^{\mathrm{EXP}}(298 \mathrm{~K})$ & 0.88 & 0.63 & 0.66 & 0.58 \\
$\sigma_{\mathrm{E}}^{\mathrm{MD}}(500 \mathrm{~K})$ & 6.6 & 5.8 & 5.2 & 3.4 \\
$\sigma_{\mathrm{NE}}^{\mathrm{MD}}(500 \mathrm{~K})$ & 8.5 & 6.6 & 6.6 & 4.1 \\
$\Lambda_{\mathrm{d}}^{\mathrm{MD}}(500 \mathrm{~K})(\mathrm{MD})$ & 0.78 & 0.88 & 0.78 & 0.83 \\
& & & &
\end{tabular}

To further shed light on the microscopic mechanisms involved in the dynamics of the ionic liquid-based electrolyte $\mathrm{LiBF}_{4} / \mathrm{BMIMBF} 4$, the ion cross dynamics was estimated by measuring pairing time between two ions. ${ }^{83}$ More in detail, we used the correlation function $P(t)=$ $\left\langle\theta(t) \theta(0)>\right.$ where $\theta(t)=1$ if both ions are aggregated at time $t$ and $\langle\theta(t)\rangle=0$ otherwise. ${ }^{84}$ As introduced above, two ions are considered aggregated if they are separated by a distance smaller than the first minimum in the $G(r)$ pair correlations presented in the Supporting Information. The distance criterion corresponds to $4.6 \AA$ for $\mathrm{Li}^{-} \mathrm{BF}_{4}$ ion pairing and $7 \AA$ for $\mathrm{Li}-\mathrm{Li}$ and $\mathrm{BMIMBF}_{4}$ ion pairing. The pairing time distributions $P(t)$ are shown in Fig. 9 for various Li contents $f$. The 
different datasets correspond to cation/anion pairing $\left(\mathrm{Li}-\mathrm{BF}_{4}\right.$ and $\left.\mathrm{BMIM}-\mathrm{BF}_{4}\right)$ as well as the $\mathrm{Li} / \mathrm{Li}$ homo pairing. While the former provides information on the typical anion/cation association lifetime, the latter sheds light on typical Li cluster lifetime. For each ion pairing type, Fig. 9 also shows the typical pairing time $\tau_{P}$ which is obtained as the time for which $P(t)=0.5$. For a given $f, \tau_{\mathrm{P}}$ is much longer for $\mathrm{Li}-\mathrm{BF}_{4}$ than $\mathrm{BMIM}-\mathrm{BF}_{4}$, therefore revealing a stronger association with the alkali cation. This result is consistent with the limited $\mathrm{Li}^{+}$mobility as can be inferred from the experimentally measured and simulated self-diffusion coefficients (see discussion above). The stability of Li based aggregates $\mathrm{Li}_{\mathrm{n}}\left[\left(\mathrm{BF}_{4}\right)_{\mathrm{m}}\right]^{(\mathrm{m}-\mathrm{n})-}$ is responsible to the longer lifetime observed for $\mathrm{Li}^{-\mathrm{BF}_{4}}$ ion and Li-Li ion pairing. The stability of such negatively charged multianions was also recently discussed in the frame of apparent negative effective Li transference numbers in similar

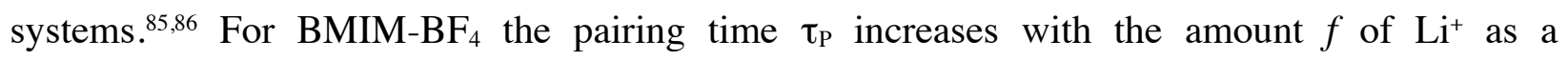
consequence of the slower dynamics as reflected from the decrease of coefficient diffusion (i.e. viscosity, not determined in this study). Conversely, the stability over time of lithium association schemes $\left(\mathrm{LiBF}_{4}\right.$ and $\mathrm{Li}-\mathrm{Li}$ based clusters) decreases as a function of Li content. This result can be explained by the fact that Li clusters appear and disappear with a frequency that increases with the Li content $f$. 


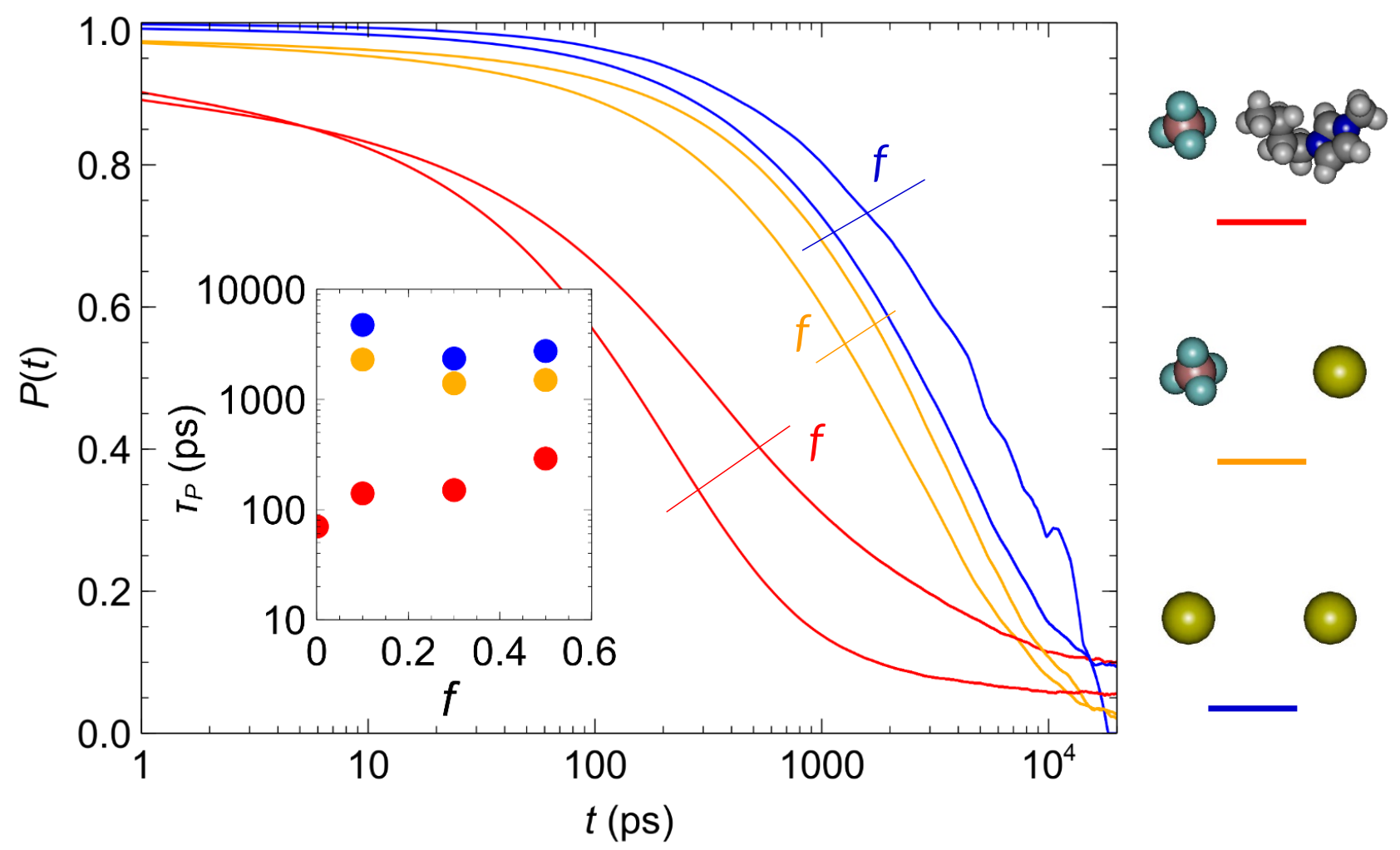

Fig. 9. Time correlation function $P(t)$ of ion/ion aggregation dynamics as estimated using molecular dynamics for a $\mathrm{BMIM} / \mathrm{BF}_{4}$ ionic liquid containing a Li cation fraction $f=0.1$ and $f=$ 0.5. Different typical pairings are shown as indicated in the graph: $\mathrm{Li} / \mathrm{BF}_{4}$ (orange), $\mathrm{BMIM} / \mathrm{BF}_{4}$ (red), and $\mathrm{Li} / \mathrm{Li}$ (blue). Ion pairing is defined for ions within a distance of $4.6 \AA$ for $\mathrm{Li}^{-\mathrm{BF}_{4}}$ and 7 $\AA$ for $\mathrm{BMIM}-\mathrm{BF}_{4}$ and $\mathrm{Li}-\mathrm{Li}$ couples. The insert shows the evolution of the pairing time $\tau_{P}$ as a function of the ratio $f$.

\section{Conclusion}

Ionic liquid-based electrolytes are considered as promising candidates for electrochemical and electrokinetic devices. Yet, in spite of being composed of ions only, they exhibit an intriguing low ionic conductivity which remains to be rationalized.$^{87,88,89,90}$ In order to get insights into the complex interplay of the structure and dynamics in these electrolytes, this paper presents a 
microscopic study using a prototypical ionic liquid-based electrolyte obtained by dissolving $\mathrm{LiBF}_{4}$ in $\mathrm{BMIMBF}_{4}$. More in detail, our strategy relies on a dual approach that includes a combination of experimental techniques (WAXS, PFG-NMR, EIS) and an all-atom MD approach to decipher the structural and dynamical properties in these complex fluids. Our analysis provides evidence that replacing a large BMIM cation by a small alkali cation leads to significant nanostructuration inside the liquid (with the appearance of a prepeak in the wide angle scattering data that becomes more pronounced upon increasing the Li concentration). Such significant positional ordering at the nanoscale is concomitant with a strong mobility hindering together with an unexpected low lithium transference number.

The force field parameters used in our our molecular dynamics are found to qualitatively capture the overall behavior of these complex mixtures. However, to reach relevant and accurate results with reasonably available CPU times, these molecular simulations are performed at higher temperatures than real-life experiments. Yet, our attempts to get results at room temperatures indicate that the qualitative picture emerging from our approach in terms of structural and dynamical aspects is virtually unchanged (as was also reported in previous studies) $)^{30,38,48,51,52}$ as it is shown for the MSD (Fig. S2) and the total structure factors (Fig. S8) of $\mathrm{BMIMBF}_{4} / \mathrm{LiBF}_{4}(f=0$ and 0.5$)$. On the other hand, in agreement with previous works ${ }^{79,91}$, the dynamical properties inferred from our molecular simlation approach underestimate the experimental data. Such a drawback could be compensated by using ion charge lower than unity, i.e. $+/-0.7^{92}$, a polarizable forcefield ${ }^{93}$ which includes polarizability contributions ${ }^{94}$ at the cost of drastically larger computing times (or a coarse grained approach which is then lacking details of the atomic molecular description).$^{95}$ However, in all cases, dimensionless transport numbers (lithium transference and dissociation ratio) are found to be well reproduced by our numerical approach. 
From a general viewpoint, this study illustrates how molecular simulation tools provide essential insights to help rationalize experimental results. In particular, X-ray or neutron scattering data in the form of $S(Q)$ curves can be deciphered using pair correlation functions $G_{i j}(r)$ and partial structure factors $S_{i j}(Q)$ available using molecular dynamics. Other microscopic features that can be unraveled using molecular dynamics include special coordination frames of $\mathrm{Li}^{+}$cations by $\mathrm{BF}_{4}^{-}$anions but also Li-based dimers, trimers and bigger aggregates that form in the electrolyte upon increasing the Li content. Such insights also provide key information about the Li solubility limit inside these mixed salts as the result of hindered ion mobility. This is also a direct consequence of the desired Li coordination (4 anion per lithium) with respect to the number of available $\mathrm{BF}_{4}^{-}$anion (1 anion per lithium). Our molecular dynamics approach also allows studying the dynamical aspects over a large time scale to probe mutiscale mobility processes. Of key relevance to the present study, MSD data from ps to ns are related to nonGaussian mobility mechanisms. ${ }^{96}$ Such mechanisms have been already identified by means of short-time dynamical experiments such as inelastic neutron scattering (density of states, vibrational dynamics) and longer time experiments such as quasielastic neutron scattering and neutron spin echo (translational mobility)..$^{39,49,68-72}$ Finally, beyond a semi-quantitative description of experimental results, molecular dynamics allows gaining fundamental insights into molecular aspects such as elementary dynamical steps, cross-correlated motion modes, molecular ion pairing, etc. Such dual approaches are thought to be essential in the design of ionic liquid-based electrolytes with optimal properties.

\section{Supporting Information Available}

The supporting information is available free of charge at http://

- Details of molecular dynamics mehodology : nomenclature used for identification of atoms, partial charge and Lennard Jones used for each atoms in the force field parameters and composition of the simulation boxes. 
- Evolution of mean square displacement with temperature $(300 \mathrm{~K}$ to $500 \mathrm{~K})$ for $\mathrm{BMIMBF}_{4}$, $\mathrm{LiBF}_{4}(f=0$ and 0.5$)$.

- partial radial distribution function $G_{i j}(r)$ and structure factor $S_{i j}(Q)$ for N-N, N-Li, $\mathrm{C}_{\mathrm{s}}-\mathrm{Li}$ and Li-Li pairs.

- Determination of the number of neighbors as obtained by integration of the COM $G_{A B}(r)$.

- Structure factors $S(Q)$ for $\mathrm{BMIMBF}_{4}, \mathrm{LiBF}_{4}(f=0$ and 0.5$)$ at $\mathrm{T}=300 \mathrm{~K}$ and $500 \mathrm{~K}$.

\section{Acknowledgments}

We acknowledge the Centre Informatique National de l'Enseignement Supérieur (CINES) for a generous supercomputer allocation (project: A0040807695). We also thank the support from the program "Investissements d'Avenir" LabEx PALM (Project ANR-10-LABX-0039-PALM). We want also to thanks Dr. Pawel Wzietek and the group ELINSTRU@LPS for their help with the development of the LPS NMR spectrometer.

\section{Bibliography}


1 Marcus, Y. Ionic Liquid Properties : from Molten Salts to RTILs; Springer, 2016.

2 Hayes, R.; Warr, G. G.; Atkin, R. Structure and nanostructure in ionic liquids. Chem. Rev. 2015, 115, 6357-6426.

3 Shi, R.; Wang, Y. Dual ionic and organic nature of ionic liquids Sci. Rep. 2016, 6, 19644.

4 Olivier-Bourbigou, H; Magna, L.; Morvan, D. Ionic Liquids and Catalysis: Recent Progress from Knowledge to Applications. Applied Catalysis A: General 2010, 373, 1-56.

5 Wilson, M.; Madden, P. A. "Prepeaks" and "First Sharp Diffraction Peaks" in Computer Simulations of Strong and Fragile Ionic Liquids. Phys. Rev. Lett. 1994, 72, 3033-3036.

6 Triolo, A.; Russina, O.; Bleif, H.-J.; Di Cola, E. Nanoscale Segregation in Room Temperature Ionic Liquids. J. Phys. Chem. B 2007, 111, 4641-4644.

7 Martinelli, A.; Maréchal, M.; Östlund, Å.; Cambedouzou, J. Insights into the Interplay Between Molecular Structure and Diffusional Motion in 1-Alkyl-3-methylimidazolium Ionic Liquids: a Combined PFG NMR and X-ray Scattering Study. Phys. Chem. Chem. Phys., 2013, 15, 5510-5517.

8 Hollócki, O.; Malberg, F.; Welton, T.; Kirchner, B. On the Origin of Ionicity in Ionic Liquids. Ion Pairing versus Charge Transfer. Phys. Chem. Chem. Phys. 2014, 16, 16880-16890.

9 Smiglak, M.; Pringle, J. M.; Lu, X.; Han, L.; Zhang, S.; Gao, H.; MacFarlane, D. R.; Rogers, R. D. Ionic Liquids for Energy, Materials, and Medicine. Chem. Commun. 2014, 50, 9228-9250.

10 Welton, T. Ionic Liquids: a Brief History. Biophys. Rev. 2018, 10, 691-706.

11 Vioux, A.; Coasne, B. From Ionogels to Biredox Ionic Liquids: Some Emerging Opportunities for Electrochemical Energy Storage and Conversion Devices. Adv. Energy Mater. 2017, 7, 1700883.

12 Watanabe, M.; Thomas, M. L.; Zhang, S.; Ueno, K.; Yasuda, T.; Dokko, K. Application of Ionic Liquids to Energy Storage and Conversion Materials and Devices. Chem. Rev. 2017, 117, 7190-7239.

13 Armand, M.; Endres, F.; MacFarlane, D. R.; Ohno, H.; Scrosati, B. Ionic-Liquid Materials for the Electrochemical Challenges of the Future. Nat. Mater. 2009, 8, 621-629.

14 Phung Le, M. L.; Alloin, F.; Strobel, P.; Leprêtre, J.-C.; Pérez del Valle, C.; Judeinstein, P. Structure-Properties Relationships of Lithium Electrolytes Based on Ionic Liquid. J. Phys. Chem. B 2010, 114, 894-903.

15 Castiglione, F.; Ragg, E.; Mele, A.; Appetecchi, G. B.; Montanino, M.; Passerini, S. Molecular Environment and Enhanced Diffusivity of $\mathrm{Li}^{+}$Ions in Lithium-Salt-Doped Ionic Liquid Electrolytes. J. Phys. Chem. Lett. 2011, 2, 153157.

16 Martin, P.-A.; Chen, F.; Forsyth, M.; Deschamps, M.; O’Dell, L. A. Correlating Intermolecular Cross-Relaxation Rates with Distances and Coordination Numbers in Ionic Liquids. J. Phys. Chem. Lett. 2018, 9, 7072-7078.

17 Gabl, S.; Steinhauser, O.; Weingärtner, H. From Short-Range to Long-Range Intermolecular NOEs in Ionic Liquids: Frequency Does Matter. Angew. Chem. Int. Ed. 2013, 52, 9242-9246.

18 Di Petro, M. E.; Castiglione F.; Mele A. J. Phys. Chem. B 2020, 124, 2879-2891.

19 Chen, F.; Howlett, P.; Forsyth, M. Na-Ion Solvation and High Transference Number in Superconcentrated Ionic Liquid Electrolytes: a Theoretical Approach. J. Phys. Chem. C 2018, 122, 105-114.

20 Lassègues, J.-C.; Grondin, J.; Talaga, D. Lithium Solvation in Bis(trifluoromethanesulfonyl)imide-Based Ionic Liquids. Phys. Chem. Chem. Phys. 2006, 8, 5629-5632.

21 Paschoal, V. H.; Faria, L. F. O.; Ribeiro, M. C. C. Vibrational Spectroscopy of Ionic Liquids. Chem. Rev., 2017, 117, 7053-7112.

22 Zhou, Q.; Boyle, P. D.; Malpezzi, L.; Mele, A.; Shin, J.-H.; Passerini, S.; Henderson, W. A. Phase Behavior of Ionic Liquid-LiX Mixtures: Pyrrolidinium Cations and TFSI- Anions - Linking Structure to Transport Properties. Chem. Mater. 2011, 23, 4331-4337.

23 Wang, Y.-L.; Li, B.; Sarman, S.; Mocci, F.; Lu, Z.-Y.; Yuan, J.; Laaksonen, A.; Fayer, M. D. Microstructural and Dynamical Heterogeneities in Ionic Liquids. Chem. Rev. 2020, 120, 5798-5877. 
24 Zhang, Y.; Maginn, E. J. Direct Correlation Between Ionic Liquid Transport Properties and Ion Pair Lifetimes: a Molecular Dynamics Study. J. Phys. Chem. Lett. 2015, 6, 700-705.

25 Dommert, F.; Wendler, K.; Berger, R.; Delle Site, L.; Holm, C. Force Fields for Studying the Structure and Dynamics of Ionic Liquids: a Critical Review of Recent Developments. ChemPhysChem 2012, 13, 1625-1637.

26 Bhargava, B. L.; Balasubramanian, S.; Klein, M. L. Modelling Room Temperature Ionic Liquids. Chem. Commun. 2008, 3339-3351.

27 Köddermann, T.; Paschek, D.; Ludwig, R. Molecular Dynamic Simulations of Ionic Liquids: a Reliable Description of Structure, Thermodynamics and Dynamics. ChemPhysChem 2007, 8, 2464-2470.

28 Köddermann, T.; Fumino, K.; Ludwig, R.; Canongia Lopes, J. N.; Pádua, A. A. H. What Far-Infrared Spectra Can Contribute to the Development of Force Fields for Ionic Liquids used in Molecular Dynamics Simulations. ChemPhysChem 2009, 10, 1181-1186.

29 Annapureddy, H. V. R.; Kashyap, H. K.; De Biase P. M.; Margulis, C. J. What is the Origin of the Prepeak in the XRay Scattering of Imidazolium-Based Room-Temperature Ionic Liquids? J. Phys. Chem. B 2010, 114, 16838-16846.

30 Kashyap, H. K.; Santos, C. S.; Annapureddy, H. V. R.; Murthy, N. S.; Margulis, C. J.; Castner, E. W. TemperatureDependent Structure of Ionic Liquids: X-Ray Scattering and Simulations. Faraday Discuss. 2012, $154,133-143$.

31 Huang, Q.; Lourenco, T. C.; Costa, L. T.; Zhang, Y.; Maginn, E. J.; Gurkan, B. Solvation Structure and Dynamics of $\mathrm{Li}^{+}$in Ternary Ionic Liquid- Lithium Salt Electrolytes. J. Phys. Chem. B 2019, 123, 516-527.

32 Haskins, J. B.; Bennett, W. R.; Wu, J. J.; Hernández, D. M.; Borodin, O.; Monk, J. D.; Bauschlicher Jr., C. W.; Lawson, J. W. Computational and Experimental Investigation of Li-Doped Ionic Liquid Electrolytes: [pyr14][TFSI], [pyr13][FSI], and [EMIM][BF 4 . J. Phys. Chem. B 2014, 118, 11295-11309.

33 Solano, C. J. F.; Jeremias, S.; Paillard, E.; Beljonne, D.; Lazzaroni, R. A Joint Theoretical/Experimental Study of the Structure, Dynamics, and $\mathrm{Li}^{+}$Transport in Bis([tri]fluoro[methane]sulfonyl)imide [T]FSI-Based Ionic Liquids. J. Chem. Phys. 2013, 139, 034502(1-15).

34 Tong, J.; Wu. S; von Solms, N.; Liang, X.; Huo, F.; Zhou, Q.; He H.; Zhang S. The Effect of Concentration of Lithium Salt on the Structural and Transport Properties of Ionic Liquid-Based Electrolytes. Frontiers in Chemistry, 2020, 7 , 945(1-10).

35 Monteiro, M. J.; Bazito, F. F. C.; Siqueira, L. J. A.; Ribeiro, M. C. C.; Torresi, R. M. Transport Coefficients, Raman Spectroscopy, and Computer Simulation of Lithium Salt Solutions in an Ionic Liquid. J. Phys. Chem. B 2008, 112, 2102-2109.

36 Fujii, K.; Hamano, H.; Doi, H.; Song, X.; Tsuzuki, S.; Hayamizu, K.; Seki, S.; Kameda, Y.; Dokko, K.; Watanabe, M.; Umebayashi, Y.; Unusual $\mathrm{Li}^{+}$Ion Solvation Structure in Bis(fluorosulfonyl)amide Based Ionic Liquid. J. Phys. Chem. C 2013, 117, 19314-19324.

37 Mao, X.; Brown, P.; Červinka, C.; Hazell, G.; Li, H.; Ren, Y.; Chen, D.; Atkin, R.; Eastoe, J.; Grillo, I.; Padua, A. A. H.; Costa Gomes, M. F.; Hatton, T. A. Self-Assembled Nanostructures in Ionic Liquids Facilitate Charge Storage at Electrified Interfaces. Nat. Mater. 2019, 18, 1350-1357.

38 Merlet, C.; Rotenberg, B.; Madden, P. A.; Taberna, P.-L.; Simon, P.; Gogotsi, Y.; Salanne, M. On the Molecular Origin of Supercapacitance in Nanoporous Carbon Electrodes. Nat. Mater. 2012, 11, 306-310.

39 Osti, N. C.; Mamontov, E. Microscopic Dynamics in Room-Temperature Ionic Liquids Confined in Materials for Supercapacitor Applications. Sustainable Energy Fuels, 2020, 4, 1554-1576.

40 Price W. S. NMR Studies of Translational Motion: Principles and Applications; Cambridge University Press, 2009.

41 Taché, O.; Rouzière, S.; Joly, P.; Amara, M.; Fleury, B.; Thill, A.; Launois, P.; Spalla, O.; Abécassis, B. MOMAC: a SAXS/WAXS Laboratory Instrument Dedicated to Nanomaterials. J. Applied Cryst. 2016, 49, 1624-1631.

42 Plimpton, S. Fast Parallel Algorithms for Short-Range Molecular Dynamics. J. Comput. Phys. 1995, 117, 1-19.

43 Canongia Lopes, J. N.; Deschamps, J.; Padua, A.A.H. Modeling Ionic Liquids using a Systematic All-Atom Force Field. J. Phys. Chem. B 2004, 108, 2038-2047.

44 Wu, X.; Liu, Z.; Huang, S.; Wang, W. Molecular Dynamics Simulation of Room-Temperature Ionic Liquid Mixture of $[\mathrm{bmim}]\left[\mathrm{BF}_{4}\right]$ and Acetonitrile by a Refined Force Field. Phys. Chem. Chem. Phys. 2005, 7, 2771-2779. 
45 Jensen, K. P.; Jorgensen, W. L. Halide, Ammonium and Alkali Metal Ion Parameters for Modeling Aqueous Solutions. J. Chem. Theory Comput. 2006, 2, 1499-1509.

46 Coasne, B.; Viau, L.; Vioux, A. Loading-Controlled Stiffening in Nanoconfined Ionic Liquids. J. Phys. Chem. Lett. 2011, 10, 1150-1154.

47 Nosé, S. A Unified Formulation of the Constant Temperature Molecular Dynamics Methods. J. Chem. Phys. 1984, 81, 511-519.

48 Merlet, C.; Salanne, M.; Rotenberg, B. New Coarse-Grained Models of Imidazolium Ionic Liquids for Bulk and Interfacial Molecular Simulations", J. Phys. Chem. C, 2012, (116), 7687-7693.

49 Osti, N. C.; Van Aken, K. L.; Thompson M. W.; Tiet F.; Jiang, D.-e.; Cummings, P. T.; Gogotsi, Y.; Mamontov, E. Solvent Polarity Governs Ion Interactions and Transport in a Solvated Room-Temperature Ionic Liquid. J. Phys. Chem. Lett. 2017, 8, 167-171.

50 Ray, P.; Balducci, A.; Kirchner B. Molecular Dynamics Simulations of Lithium-Doped Ionic-Liquid Electrolytes, J. Phys. Chem. 2018, 122, 10535-10547.

51 Araque, J. C.; Yadav, S. K.; Shadeck, M.; Maroncelli, M.; Margulis, C. J. How is Diffusion of Neutral and Charged Tracers Related to the Structure and Dynamics of a Room-Temperature Ionic Liquid? Large Deviations from Stokes-Einstein Behavior Explained. J. Phys. Chem. B 2015, 119, 7015-7029.

52 Rey-Castro, C.; Vega, L. F. Transport Properties of the Ionic Liquid 1-Ethyl-3-methylimidazolium Chloride from Equilibrium Molecular Dynamics Simulation. The effect of Temperature. J. Phys. Chem. B 2006, 110, 14426-14435.

53 Aguilera, L.; Völkner, J.; Labrador, A.; Matic, A. The Effect of Lithium Salt Doping on the Nanostructure of Ionic Liquids. Phys. Chem. Chem. Phys. 2015, 17, 27082-27087.

54 Araque, J. C.; Hettige, J. J.; Margulis, C. J. Modern Room-Temperature Ionic Liquids, a Simple Guide to Understanding their Structure and How it May Relate to Dynamics. J. Phys. Chem. C 2015, 119, 12727-12740.

55 Pilar, K.; Balédent, V.; Zeghal, M.; Judeinstein, P.; Jeong, S.; Passerini, S.; Greenbaum, S. Investigation of Ion Aggregation in Ionic Liquids and their Solutions with Lithium Salt Under High Pressure. J. Chem. Phys. 2018, 148, 031102 .

56 Méndez-Morales, T.; Carrete, J.; Bouzón-Capelo, S.; Pérez-Rodríguez, M.; Cabeza, O.; Gallego, L. J.; Varela, L. M. MD Simulatons of the Formation of Stable Clusters in Mixtures of Alkaline Salts and Imidazolium-Based Ionic Liquids. J. Phys. Chem. B 2013, 117, 3207-3220.

57 Tokuda, H.; Hayamizu, K.; Ishii, K.; Bin Hasan Susan, Md. A.; Watanabe, M. Physicochemical Properties and Structures of Room Temperature Ionic Liquids. 1. Variation of Anionic Species. J. Phys. Chem. B 2004, 108, 1659316600 .

58 Harris, K. R.; Kanakubo, M. Revised and Extended Values for Self-Diffusion Coefficients of 1-alkyl-3Methylimidazolium Tetrafluoroborates and Hexafluorophosphates: Relations Between the Transport Properties. J. Phys. Chem. B 2016, 120, 12937-12949.

59 Kruk, D.; Meier, R.; Rachocki, A.; Korpala, A.; Singh, R. K.; Rössler A. Determining Diffusion Coefficients of Ionic Liquids by Means of Field Cycling Nuclear Magnetic Resonance Relaxometry. J. Chem. Phys. 2014, 140, 244509.

60 Hayamizu, K.; Aihara, Y.; Nakagawa, H.; Nukuda, T.; Price, W. S. Ionic Conduction and Ion Diffusion in Binary Room-Temperature Ionic Liquids Composed of $[\mathrm{emim}]\left[\mathrm{BF}_{4}\right]$ and $\mathrm{LiBF}_{4}$. J. Phys. Chem. B 2004, 108, 19527-19532.

61 Wang, H.; Liu, S.; Huang, K.; Yin, X.; Liu, Y.; Peng, S. BMIMBF 4 Ionic Liquid Mixtures Electrolyte for Li-Ion Batteries. Int. J. Electrochem. Sci. 2012, 7, 1688-1698.

62 Keen, D. A. A Comparison of Various Commonly Used Correlation Functions for Describing Total Scattering. J. Appl. Cryst. 2001, 34, 172-177.

63 Ashcroft, N. W.; Langreth, D. C. Structure of Binary Liquid Mixtures. I. Phys. Rev. 1967, 156, 685-692.

64 Liu, H., Maginn, E. Effect of Ion Structure on Conductivity in Lithium-Doped Ionic Liquid Electrolytes: a Molecular Dynamics Study. J. Chem. Phys. 2013, 139, 114508.

65 Seo, D. M.; Boyle, P. D.; Allen, J. L.; Han, S.-D.; Jonsson, E.; Johansson, P.; Henderson, W. A. Solvate Structures and Computational/Spectroscopic Characterization of $\mathrm{LiBF}_{4}$ Electrolytes. J. Phys. Chem. C 2014, 118, $18377-18386$. 
66 Kashyap, H. K.; Hettige, J. J.; Annapureddy, H. V. R.; Margulis, C. J. SAXS Anti-Peaks Reveal the Length-Scales of Dual Positive-Negative and Polar-Apolar Ordering in Room-Temperature Ionic liquids. Chem. Commun. 2012, 48, 5103-5105.

67 Olsher, U.; Izatt, R. M.; Bradshaw, J. S.; Dalley, N. K. Coordination Chemistry of Lithium Ion: a Crystal and Molecular Structure Review. Chem. Rev. 1991, 91, 137-164.

68 Ferdeghini, F.; Berrod, Q.; Zanotti, J.-M.; Judeinstein, P.; Garcia Sakai, V.; Czakkel, O.; Fouquet, P.; Constantin, D. Nanostructuration of Ionic Liquids: Impact on the Cation Mobility. A Multi-Scale Study. Nanoscale 2017, 9, 19011908.

69 Berrod, Q.; Ferdeghini, F.; Zanotti, J.-M.; Judeinstein, P.; Lairez, D.; Garcia Sakai, V.; Czakkel, O.; Fouquet, P.; Constantin, D. Ionic Liquids: Evidence of the Viscosity Scale-Dependence. Sci. Rep. 2017, 7, 2241.

70 Kofu, M.; Nagao, M.; Ueki, T.; Kitazawa, Y.; Nakamura, Y.; Sawamura, S.; Watanabe, M.; Yamamuro, O.; Heterogeneous Slow Dynamics of Imidazolium-Based Ionic Liquids Studied by Neutron Spin Echo. J. Phys. Chem. B 2013, 117, 2773-2781.

71 Jafta, C. J.; Bridges, C.; Haupt, L.; Do, C.; Sippel, P.; Cochran, M. J.; Krohns, S.; Ohl, M.; Loidl, A.; Mamontov, E.; Lunkenheimer, P.; Dai, S.; Sun, X.-G. Ion Dynamics in Ionic-Liquid-Based Li-Ion Electrolytes Investigated by Neutron Scattering and Dielectric Spectroscopy. ChemSusChem 2018, 11, 3512.

72 Panesar, K. S.; Judeinstein P.; Zanotti, J.-M. Selective Deuteration Reveals Interference Caused by Sidechain Dynamics on Measurements of Self-Diffusion in Ionic Liquid Cations. J. Phys. Soc. Jpn., 2013, 82, SA013(1-9).

73 Hayamizu, K.; Tsuzuki, S.; Seki, S.; Umebayashi, Y. Multinuclear NMR Studies on Translational and Rotational Motion for Two Ionic Liquids Composed of $\mathrm{BF}_{4}$ Anion. J. Phys. Chem. B 2012, 116, 11284-11291.

74 Prabhu, S. R.; Dutt, G. B. Rotational Diffusion of Nonpolar and Ionic Solutes in 1-Alkyl-3-methylimidazolium Tetrafluoroborate-LiBF 4 Mixtures: Does the Electrolyte Induce Structure-Making or Structure-Breaking Effect? J. Phys. Chem. B 2015, 119, 15040-15045.

75 Casalegno, M.; Raos, G.; Appetecchi, G. B.; Passerini, S.; Castiglione, F.; Mele, A. From Nanoscale to Microscale: Crossover in the Diffusion Dynamics within two Pyrrolidinium-Based Ionic Liquids. J. Phys. Chem. Lett. 2017, 8, 5196-5202.

76 Schmidt, D. A.; Birer, O.; Funkner, S.; Born, B. P.; Gnanasekaran, R.; Schwaab, G. W.; Leitner, D. M.; Havenith, M. Rattling in the Cage: Ions as Probes of Sub-Picosecond Water Network Dynamics. J. Am. Chem. Soc. 2009, 131, $18512-18517$.

77 Postupna, O. O.; Kolesnik, Y. V.; Kalugin, O. N.; Prezhdo, O. V. Microscopic Structure and Dynamics of LiBF 4 Solutions in Cyclic and Linear Carbonates. J. Phys. Chem. B 2011, 115, 14563-1457.

78 Chang, S.; Schmidt, P. P.; Severson, M. W. Far-Infrared Spectrum of Solvated Lithium Cations in Dimethyl Sulfoxide. J. Phys. Chem. 1986, 90, 1046-1050.

79 Picálek, J.; Kolafa, J.; Molecular Dynamics Study of Conductivity of Ionic Liquids: The Kohlrausch Law. J. Mol. Liq. 2007, 134, 29-33.

80 Borodin, O.; Smith, G. D. Structure and Dynamics of N-Methyl-N-propylpyrrolidinium Bis(trifluoromethane sulfonyl) imide Ionic Liquid from Molecular Dynamics Simulations. J. Phys. Chem. B 2006, 110, 11481-11490.

81 Liu, H.; Maginn, E. An MD Study of the Applicability of the Walden Rule and the Nernst-Einstein Model for Ionic Liquids. ChemPhysChem 2012, 13, 1701-1707.

82 Afandak, A.; Eslami, H. Ion-Pairing and Electrical Conductivity in the Ionic Liquid 1-n-Butyl-3-methylimidazolium Methylsulfate [Bmim][MeSO 4 ]: Molecular Dynamics Simulation Study. J. Phys. Chem. B 2017, 121, 7699-7708.

83 Zhao, W.; Leroy, F.; Heggen, B.; Zahn, S.; Kirchner, B.; Balasubramanian, S.; Müller-Plathe, F. Are there Stable IonPairs in Room-Temperature Ionic Liquids? Molecular Dynamics Simulations of 1-n-Butyl-3-methylimidazolium Hexafluorophosphate. J. Am. Chem. Soc. 2009, 131, 15825-15833.

84 Cazade, P. -A.; Hartkamp, R.; Coasne, B. Structure and Dynamics of an Electrolyte Confined in Charged Nanopores. J. Phys. Chem. C 2014, 118, 5061-5072.

85 Gouverneur, M.; Schmidt, F.; Schönhoff, M. Negative Effective Li Transference Numbers in Li Salt/Ionic Liquid Mixtures: Does Li Drift in the "Wrong" Direction. Phys. Chem. Chem. Phys. 2018, 20, 7470-7478. 
86 Harris, K. R. Can the Transport Properties of Molten Salts and Ionic Liquids be Used to Determine Ion Association? Phys. Chem. B 2016, 120, 12135-12147.

87 Xu, W.; Cooper, E. I.; Angell, C. A. Ionic Liquids: Ion Mobilities, Glass Temperatures, and Fragilities. J. Phys. Chem. B 2003, 107, 6170-6178.

88 Lee, A. A.; Vella, D.; Perkin, S.; Goriely, A. Are Room-Temperature Ionic Liquids Dilute Electrolytes? J. Phys. Chem. Lett. 2015, 6, 159-163.

89 MacFarlane, D. R.; Forsyth, M.; Izgorodina, E. I.; Abbott, A. P.; Annat, G.; Fraser, K. On the Concept of Ionicity in Ionic Liquids. Phys. Chem. Chem. Phys. 2009, 11, 4962-4967.

90 Ueno, K.; Tokuda, H.; Watanabe, M. Ionicity in Ionic Liquids: Correlation with Ionic Structure and Physicochemical Properties. Phys. Chem. Chem. Phys. 2010, 12, 1649-1658.

91 Tsuzuki, S.; Shinoda, W.; Saito, H.; Mikami, M.; Tokuda, H.; Watanabe, M. Molecular Dynamics Simulations of Ionic Liquids: Cation and Anion Dependence of Self-Diffusion Coefficients of Ions. J. Phys. Chem. B, 2009, 113, 1064110649.

92 Schröder, C. Comparing Reduced Partial Charge with Polarizable Simulations of Ionic Liquids. Phys. Chem. Chem. Phys. 2012, 14, 3089-3102.

93 Cazade, P.-A.; Dweik, J.; Coasne, B.; Henn, F.; Palmeri, J. Molecular Simulation of Ion-Specific Effects in Confined Electrolyte Solutions using Polarizable Forcefields. J. Phys. Chem. C 2010, 114, 12245-12257.

94 Bedrov, D.; Piquemal, J.-P.; Borodin, O.; MacKerell Jr., A. D.; Roux, B.; Schröder, C. Molecular Dynamics Simulations of Ionic Liquids and Electrolytes using Polarizable Force Fields. Chem. Rev. 2019, 119, 7940-7995.

95 Salanne, M. Simulations of Room Temperature Ionic Liquids: from Polarizable to Coarse-Grained Force Fields. Phys. Chem. Chem. Phys. 2015, 17, 14270-14279.

96 Araque, J. C.; Hettige, J. J.; Margulis, C. J. Ionic Liquids - Conventional Solvent Mixtures, Structurally Different but Dynamically Similar. J. Chem. Phys. 2015, 143, 134505 